\title{
Construction of a risk prediction model using m6A RNA methylation regulators in prostate cancer: comprehensive bioinformatic analysis and histological validation
}

Yongjun Quan ${ }^{1}$, Xiaodong Zhang ${ }^{3}$ and Hao Ping ${ }^{1,2^{*}}$ (1)

\begin{abstract}
Background: Epigenetic reprogramming reportedly has a crucial role in prostate cancer (PCa) progression. RNA modification is a hot topic in epigenetics, and N6-methyladenosine (m6A) accounts for approximately $60 \%$ of RNA chemical modifications. The aim of this study was to evaluate the m6A modification patterns in PCa patients and construct a risk prediction model using m6A RNA regulators.

Materials and methods: Analyses were based on the levels of $25 \mathrm{~m} 6 \mathrm{~A}$ regulators in The Cancer Genome Atlas (TCGA). Differentially expressed gene (DEG) and survival analyses were performed according to TCGA-PRAD clinicopathologic and follow-up information. To detect the influences of m6A regulators and their DEGs, consensus clustering analysis was performed, and tumor mutational burden (TMB) estimation and tumor microenvironment (TME) cell infiltration were assessed. mRNA levels of representative genes were verified using clinical PCa data.

Results: Diverse expression patterns of m6A regulators between tumor and normal (TN) tissues were detected regarding Gleason score (GS), pathological T stage (pT), TP53 mutation, and survival comparisons, with HNRNPA2B1 and IGFBP3 being intersecting genes. HNRNPA2B1 was upregulated in advanced stages (GS > 7, pT3, HR > 1, and TP53 mutation), as verified using clinical PCa tissue. Three distinct m6A modification patterns were identified through consensus clustering analysis, but no significant difference was found among these groups in recurrence-free survival (RFS) analysis. Six DEGs of m6A clusters (m6Aclusters) were screened through univariate Cox regression analysis. $M M A B$ and PAIAP2 were intersecting genes for the five clinical factors. MMAB, which was upregulated in PCa compared with TN, was verified using clinical PCa samples. Three distinct subgroups were established according to the 6 DEGs. Cluster A involved the most advanced stages and had the poorest RFS. The m6A score (m6Ascore) was calculated based on the 6 genes, and the low m6Ascore group showed poor RFS with a negative association with infiltration for 16 of 23 immune-related cells.

Conclusion: We screened DEGs of m6Aclusters and identified 6 genes (BAIAP2, TEX264, MMAB, JAGN1, TIMM8AP1, and IMP3), with which we constructed a highly predictive model with prognostic value by dividing TCGA-PRAD into three distinct subgroups and performing m6Ascore analysis. This study helps to elucidate the integral effects of m6A modification patterns on PCa progression.
\end{abstract}

\footnotetext{
${ }^{*}$ Correspondence: pinghaotrh@ccmu.edu.cn

${ }^{1}$ Department of Urology, Beijing Tongren Hospital, Capital Medical University, Beijing 100730, China

Full list of author information is available at the end of the article
}

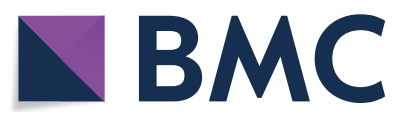

(c) The Author(s) 2022. Open Access This article is licensed under a Creative Commons Attribution 4.0 International License, which permits use, sharing, adaptation, distribution and reproduction in any medium or format, as long as you give appropriate credit to the original author(s) and the source, provide a link to the Creative Commons licence, and indicate if changes were made. The images or other third party material in this article are included in the article's Creative Commons licence, unless indicated otherwise in a credit line to the material. If material is not included in the article's Creative Commons licence and your intended use is not permitted by statutory regulation or exceeds the permitted use, you will need to obtain permission directly from the copyright holder. To view a copy of this licence, visit http://creativecommons.org/licenses/by/4.0/. The Creative Commons Public Domain Dedication waiver (http://creativeco mmons.org/publicdomain/zero/1.0/) applies to the data made available in this article, unless otherwise stated in a credit line to the data. 
Keywords: Prostate cancer, N6-methyladenosine (m6A), The Cancer Genome Atlas (TCGA), Recurrence-free survival, m6Acluster, geneCluster, m6Ascore

\section{Introduction}

Prostate cancer $(\mathrm{PCa})$ is a leading malignant tumor among men [1]. PCa has primarily been treated with surgical prostatectomy or androgen deprivation therapy (ADT). However, it can become castration-resistant $\mathrm{PCa}$ (CRPC), and biochemical recurrence or metastasis may occur during traditional therapy, which is the main cause of cancer-specific death. Therefore, elucidating the molecular mechanisms related to PCa progression is crucial in the discovery of diagnostic biomarkers and therapeutic targets.

Epigenetic reprogramming is reported to serve a crucial role in the progression of PCa [2]. Recently, RNA modification has been regarded as a hot topic in epigenetic research, and nearly 172 different RNA modifications are present in MODOMICS [3]. Among them, N6-methyladenosine (m6A) is widespread throughout the transcriptome; indeed, m6A comprises approximately $60 \%$ of RNA chemical modifications and is present on $0.1 \%$ to $0.4 \%$ of total adenosine residues, including $>300$ noncoding RNAs and 7600 mRNAs, in eukaryotes [46]. RNA m6A methylation regulates mRNA alternative splicing, stability, and intracellular localization, constituting the major posttranscriptional modification [7]. The formation of $\mathrm{m} 6 \mathrm{~A}$ is regulated by three categories of proteins: readers (which recognize m6A-modified sites, such as YTHDC1, YTHDC2, YTHDF1, YTHDF2, YTHDF3, HNRNPC, FMR1, LRPPRC, HNRNPA2B1, IGFBP1, IGFBP2, IGFBP3, RBMX, and ELAVL1), writers (methyltransferases, such as METTL3, METTL14, METTL16, WTAP, VIRMA (also called KIAA1429), ZC3H13, RBM15, RBM15B, and CBLL1), and erasers (demethylases, such as FTO and ALKBH5).

$\mathrm{m} 6 \mathrm{~A}$ regulatory genes are reported to participate in various carcinogenic and tumor progression processes $[8,9]$. METTL3 is reported to advance PCa progression and is associated with poor prognosis by stabilizing the mRNAs of MYC, LEF1, and integrin $\beta 1$ (ITGB1) by m6A methylation [10-12]. However, one study suggested that low expression of METTL3 is associated with resistance to therapy with androgen receptor antagonists via upregulation of NR5A2/LRH-1 [13]. This finding indicates the controversy and opposing functions of METTL3 in PCa. YTHDF2-induced AKT phosphorylation and MDB3B m6A modification may also promote $\mathrm{PCa}$ proliferation, migration, and invasion [14, 15]. FTO, an m6A demethylase, inhibits the invasion and migration of PCa cells by regulating total m6A levels [16]. Nevertheless, there are insufficient data on $\mathrm{m} 6 \mathrm{~A}$ regulators in $\mathrm{PCa}$, and the role of m6A regulators remains controversial; in general, comprehensive transcriptome and genomic analysis is needed. This study fully analyzed m6A-related genes in PCa progression and prognosis.

\section{Materials and methods Data acquisition}

Transcriptome profiling and single nucleotide variation data for prostate adenocarcinoma in The Cancer Genome Atlas (TCGA) were downloaded from the GDC Data Portal (https://portal.gdc.cancer.gov/). Copy number and clinical phenotype data were downloaded from the University of California Santa Cruz Xena (https://xena. ucsc.edu/). Gene expression matrices were extracted and obtained through Practical Extraction and Report Language (Perl) (version 5.34.0) and $\mathrm{R}$ software (4.0.3) ( $\mathrm{R}$ Development Core Team, Vienna, Austria). The R package "RCircos" was used to generate Circos plots.

\section{Differentially expressed gene (DEG) analysis}

mRNA levels were analyzed with TPM (transcripts per kilobase of exome per million mapped reads) data, which were transformed from the HTSeq-FPKM transcriptome profiling data of TCGA-PRAD. The R packages "limma" and "ggpurb" were then used to identify DEGs between the normal and tumor groups and for further statistical analysis. The Wilcoxon or Kruskal-Wallis test was performed to determine DEG levels, and $\mathrm{P}<0.05$ was identified as statistically significant. The $R$ packages "clusterProfiler" and "enrichplot" were used to analyze Gene Ontology (GO) and Kyoto Encyclopedia of Genes and Genomes (KEGG) pathway enrichment.

\section{Survival and correlation analyses}

Survival data for "biochemical_recurrence", "days_to_ first_biochemical_recurrence", and "days_to_last_follow_ up.diagnoses" were obtained from clinical phenotype data. When "days_to_first_biochemical_recurrence" was indicated, we regarded these patients as having "recurrence status", and the time notated was used as the "recurrence follow-up time". Other cases were regarded as having "no recurrence", and "days_to_last_follow_ up.diagnoses" was used as "recurrence_follow_up_time". The R packages "survival" and "survminer" were used for survival analysis. Survival curves were evaluated through Kaplan-Meier and log-rank tests. Correlation analysis was performed through Pearson or Spearman correlation 
analysis, and a prognostic network map was drawn using the R packages "igraph", "psych", "reshape2", and "RColorBrewer". To build a PCa prognostic model using DEGs of m6A clusters, univariate Cox regression analysis was conducted, and $\mathrm{P}<0.05$ was used for later gene consensus clustering analysis.

\section{Consensus clustering analysis and principal component analysis (PCA)}

To determine whether m6A regulators are related to $\mathrm{PCa}$ prognosis, the cohort from TCGA was allocated into different groups based on the consensus level of m6A regulators. The process was performed using the $\mathrm{R}$ package "ConsensusClusterPlus" and resulted in cluster consensus and item-consensus results. The graphical output consisted of heatmaps, consensus cumulative distribution function (CDF) plots and delta area plots. The cluster number was determined through a high consistency of clusters, a low coefficient of variation, and no significant increase in the CDF curve. The chi-square test or Fisher's exact test was used to analyze clinicopathological characteristics and clustering. The heatmap was generated through the R package "pheatmap". Recurrence-free survival (RFS) was detected among groups using KaplanMeier and log-rank tests. PCA was performed to judge the fitness of the classification with the prcomp function of R software.

\section{Gene set variation analysis (GSVA)}

GSVA is used for assessing KEGG gene set enrichment, which is a nonparametric and unsupervised method [17]. GSVA could comprehensively score the gene sets of interest and translate them into the pathway level. In this study, to evaluate the potential pathway changes of different clusters, we downloaded "KEGG gene sets as Gene Symbols" from the GSEA website (http://www. gsea-msigdb.org/gsea/) and used the GSVA algorithm to comprehensively score each gene set.

\section{Tumor mutational burden (TMB) estimation}

TMB was calculated by the total number of mutated/total covered bases [18]. PCa was classified into two groups based on the optimum threshold segmentation of TMB status population. We analyzed the relationship between the m6A score (shown as m6Ascore below) and the TMB and then performed survival analysis comparing prognosis between the high TMB and low TMB groups.

\section{Tumor microenvironment (TME) cell infiltration}

TME infiltration levels were calculated through singlesample gene set enrichment analysis (ssGSEA) and quantified using enrichment scores [17]. The gene set of each
TME infiltrating immune cell type was obtained as previously reported [19]. Correlation analysis between m6Ascore and immune-associated genes was performed to illustrate the relationship.

\section{Clinical PCa samples}

Forty-five pathologically diagnosed $\mathrm{PCa}$ patients (15 with Gleason score (GS) $<7,15$ with $G S=7$, and 15 with GS $>7$ ) were recruited from Beijing Tongren Hospital and Beijing Chaoyang Hospital in accordance with the Ethics Committee of Beijing Tongren Hospital and Beijing Chaoyang Hospital, affiliated with Capital Medical University. All patients underwent prostatectomy between 2016 and 2021; PCa and adjacent normal tissues were removed and stored in liquid nitrogen. The clinicopathological characteristics of PCa patients are shown in Table 1.

\section{Quantitative real-time PCR (qPCR) analysis}

qPCR analysis was performed as previously described by our group $[20,21]$. Total RNA was isolated using TRIzol ${ }^{\mathrm{TM}}$ reagent (Invitrogen), and complementary DNA (cDNA) was synthesized through One-Step gDNA Removal and cDNA Synthesis SuperMix (TransGen Biotech, Beijing, China). qPCR was performed using Top Green qPCR SuperMix (TransGen Biotech) on an SDS 7500 FAST Real-Time PCR system (Applied Biosystems, Foster City, CA, USA). GAPDH or 18S ribosomal RNA was used as an endogenous reference gene. The relevant primer sequences are shown in Additional file 1: Table S1.

\section{Immunophenoscore (IPS) analysis}

IPS analysis was performed as previously reported [22]. IPS determines immunogenicity by referring to effector cells, immunosuppressive cells, MHC molecules and immunomodulators. IPS (ranging from 0 to 10) was calculated according to the gene expression of the representative cell types without bias using machine learning methods. The IPS results of TCGA-PRAD patients were downloaded from The Cancer Immunome Atlas (TCIA) (https://tcia.at/home).

\section{Statistical analysis}

Statistical analyses were conducted using $\mathrm{R}$ software (4.0.3), SPSS software version 23 (IBM, Armonk, New York, USA), and GraphPad Prism 7.0 (GraphPad Software, La Jolla, CA, USA). Incomplete data were excluded. Chi-square or Fisher's exact tests were used for categorical variables, and Wilcoxon or Kruskal-Wallis tests were used for continuous data. Correlations between two levels were assessed through Pearson or Spearman correlation analysis. Survival analysis was applied through the 
Table 1 Clinicopathological characteristics of PCa patients

\begin{tabular}{|c|c|}
\hline Clinicopathological parameters & Total $(n=45)(\%)$ \\
\hline \multicolumn{2}{|l|}{ Age } \\
\hline Median (IQR) & $65(59.5-70.0)$ \\
\hline Range (Min, Max) & $52-78$ \\
\hline$<65$ & $22(48.9 \%)$ \\
\hline$\geq 65$ & $23(51.1 \%)$ \\
\hline Total PSA (t-PSA) & $(n g / m l)$ \\
\hline Median (IQR) & $\begin{array}{l}15.28 \\
(8.61-38.30)\end{array}$ \\
\hline Range (Min, Max) & $1.05-92.21$ \\
\hline$<4 \mathrm{ng} / \mathrm{ml}$ & $4(8.9 \%)$ \\
\hline $4-10 \mathrm{ng} / \mathrm{ml}$ & $12(26.7 \%)$ \\
\hline $10-20 \mathrm{ng} / \mathrm{ml}$ & $12(26.7 \%)$ \\
\hline$>20 \mathrm{ng} / \mathrm{ml}$ & $17(37.8 \%)$ \\
\hline \multicolumn{2}{|l|}{ Gleason Score (GS) } \\
\hline$<7$ & $15(33.3 \%)$ \\
\hline$=7$ & $15(33.3 \%)$ \\
\hline$>7$ & $15(33.3 \%)$ \\
\hline \multicolumn{2}{|l|}{ Clinical T-stage } \\
\hline $\mathrm{T} 2 \mathrm{a}$ & $6(13.3 \%)$ \\
\hline $\mathrm{T} 2 \mathrm{~b}$ & $12(26.7 \%)$ \\
\hline $\mathrm{T} 2 \mathrm{C}$ & $15(33.3 \%)$ \\
\hline T3a or T3b & $12(26.7 \%)$ \\
\hline \multicolumn{2}{|l|}{ Lymph node metastasis } \\
\hline No & $30(66.7 \%)$ \\
\hline N1 & $15(33.3 \%)$ \\
\hline \multicolumn{2}{|l|}{ Distant metastasis } \\
\hline Mo or Mx & $41(91.1 \%)$ \\
\hline M1 & $4(8.9 \%)$ \\
\hline \multicolumn{2}{|l|}{ TNM stage } \\
\hline$|-| \mid$ & $25(55.6 \%)$ \\
\hline III-IV & $20(44.4 \%)$ \\
\hline \multicolumn{2}{|l|}{ Log0.5 MMAB expression } \\
\hline Median (IQR) & $\begin{array}{l}13.823837 \\
(13.254977-14.245784)\end{array}$ \\
\hline Range (Min, Max) & $11.968522-15.233100$ \\
\hline \multicolumn{2}{|l|}{ Log0.5 IGFBP3 expression } \\
\hline Median (IQR) & $\begin{array}{l}12.619003 \\
(11.692416-13.321569)\end{array}$ \\
\hline Range (Min, Max) & $10.851545-14.289084$ \\
\hline \multicolumn{2}{|l|}{ Log0.5 HNRNPA2B1 expression } \\
\hline Median (IQR) & $\begin{array}{l}11.927719 \\
(11.106794-12.651593)\end{array}$ \\
\hline Range (Min, Max) & $10.123230-13.496253$ \\
\hline
\end{tabular}

log-rank test of Kaplan-Meier survival analysis and hazard ratio (HR) with the $95 \%$ confidence interval (CI) of univariate Cox proportional hazard models. $\mathrm{P}<0.05$ was indicated as significant.

\section{Results}

Characteristics of $\mathrm{m} 6 \mathrm{~A}$ regulators in $\mathrm{PCa}$

In the TCGA-PRAD dataset, we analyzed copy number variation $(\mathrm{CNV})$ analysis alterations, DEGs, and the mutation frequency of $\mathrm{m} 6 \mathrm{~A}$ regulators of $\mathrm{PCa}$ through comparison with normal samples. For $\mathrm{CNV}$ events, approximately $76 \%(19 / 25)$ of m6A regulators lost DNA copy number, with $\mathrm{ZC} 3 \mathrm{H} 14$ having the highest degree of copy number loss (28.49\%) (Fig. 1A). Six m6A regulators gained copy number, among which VIRMA had the highest such percentage (3.19\%) (Fig. 1A). The m6A regulator $\mathrm{CNV}$ alterations and locations on chromosomes are shown in the lower panel of Fig. 1A. Of the 499 tumor and 52 normal samples in the dataset from TCGA, DEGs of m6A regulators were statistically estimated using TPM data. The levels of METTL3, HNRNPA2B1, RBM15B, and IGFBP2 were higher, while those of ZC3H13, FTO, and IGFBP3 were lower in PCa tissues than in normal tissues $(\mathrm{P}<0.001)$ (Fig. $1 \mathrm{~B}$ and Table 2). In mutation frequency analyses, 16 m6A-related genes were mutated among 19 of 484 (3.93\%) samples; mutations in VIRMA (KIAA1429), YTHDC2, RBM15B, YTHDF2, and IGFBP1 were detected in one sample (Fig. 1C). ZC3H14 exhibited the highest mutation rate (4/484), and all $25 \mathrm{~m} 6 \mathrm{~A}$ regulators exhibited low mutation rates $(<1 \%)$ in TCGA samples. This suggests the relatively conserved and stable expression of $\mathrm{m} 6 \mathrm{~A}$ regulators during PCa progression.

\section{Expression of $\mathrm{m} 6 \mathrm{~A}$ regulators in $\mathrm{PCa}$ prognosis and different clinicopathological characteristics}

The expression of m6A regulators in different GSs and pathological $\mathrm{T}(\mathrm{pT})$ stages was estimated using TCGAPARD TPM data. As PCa with GS $\geq 7$ is associated with worse prognosis $[23,24]$, the PCa samples were divided into three groups: $\mathrm{GS}<7, \mathrm{GS}=7$, and GS $>7$. Compared with the GS $<7$ group, IGFBP3, HNRNPA2B, RBMX, RBM15B, YTHDF1, HNRNPC, VIRMA, and FMR1 were significantly highly expressed in the GS $>7$ group $(\mathrm{P}<0.001)$ (Fig. $2 \mathrm{~A}$ and Table 3$)$. In addition, T2 (188), T3 (295), and T4 (10) pT stages were compared. Among the 25 m6A regulators, IGFBP3, HNRNPA2B1, VIRMA, and RBMX were more highly expressed in the T3 stage than in the T2 stage $(\mathrm{P}<0.001)$ (Fig. $2 \mathrm{~B}$ and Table 4 ). Based on Kaplan-Meier curves in survival analysis, high expression of HNRNPA2B1, IGFBP1, and ELAVL1 was associated with poor RFS $(\mathrm{P}<0.001)$ (Fig. $2 \mathrm{C}$ ). Moreover, TP53 mutation in PCa was associated with shorter radiographic progression-free survival (rPFS) and time to CRPC [25]. Compared with the TP53 wild-type group of PCa samples, the levels of VIRMA and IGFBP3 were higher and the level of IGFBP2 was lower in the TP53 

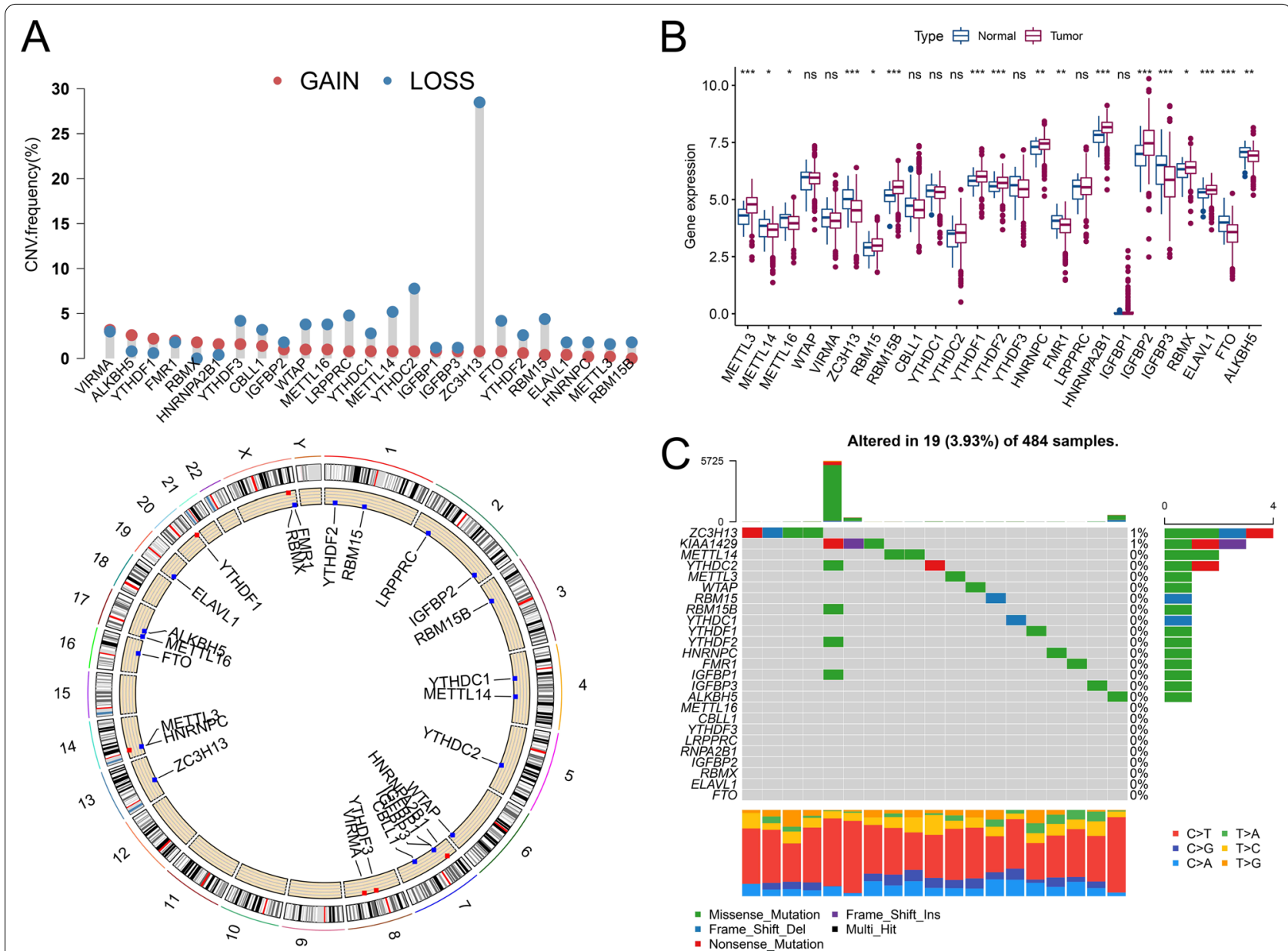

Fig. 1 Characteristics of m6A regulators in prostate cancer. A (top) The CNV variation frequency of m6A regulators in TCGA-PRAD. Blue dot: deletion frequency; red dot: amplification frequency. (bottom) The location of CNV alterations of m6A regulators on 23 chromosomes. Blue dot: copy number loss, red dot: copy number gain. B Box plot of the expression of the 25 m6A regulators in PCa and normal tissues. Median values \pm interquartile ranges are shown in the graph. $\mathrm{ns} P>0.05 ;{ }^{*} \mathrm{P}<0.05 ;{ }^{* *} \mathrm{P}<0.01$; ${ }^{* * *} \mathrm{P}<0.001$. C The mutation frequency of 25 m6A regulators in 484 TCGA-PRAD patients is shown on the waterfall plot. Columns represent individual patients, the upper bar plot shows the TMB, the right bar plot shows the proportion of each variant type, and the stacked bar plot below shows the transformed fraction of each patient

mutation group $(\mathrm{P}<0.001)$ (Fig. $2 \mathrm{D}$ and Table 5). Risk factors and relationships of m6A regulators are summarized in the prognosis network shown in Fig. 2E.

We analyzed DEGs of $\mathrm{m6A}$ regulators in GS (GS $>7$ versus (vs.) GS $<7$ ), pT (T3 vs. T2), RFS (P value of univariate Cox regression analysis $<0.05$ ), TN (tumor vs. normal $\mathrm{PCa}$ tissues), and TP53 (mutation vs. wild type) comparisons. Intersecting genes were identified via a Venn diagram, and HNRNPA2B1 and IGFBP3 were differentially expressed in all comparisons (Fig. 2F). The level of HNRNPA2B1 was higher in the PCa group than in the normal group and high in association with advanced-stage parameters: namely, GS $>7$, pT3, HR > 1, and TP53 mutation. However, the level of IGFBP3 was lower in PCa tissues but higher in the presence of the above advanced-stage indicators. This suggests that different molecular mechanisms and expression patterns may exist in $\mathrm{PCa}$ and throughout progression. We then assessed the expression of HNRNPA2B1 and IGFBP3 in 45 PCa tissues (including 15 with $\mathrm{GS}<7,15$ with GS $=7$, and 15 with GS $>7$ ) and 15 adjacent normal prostate tissues. Levels of HNRNPA2B1 and IGFBP3 were higher in $66.7 \%(10 / 15, \mathrm{P}<0.05)$ and $40.0 \%(6 / 15, \mathrm{P}>0.05)$ of $\mathrm{PCa}$ tissues than in adjacent normal tissues, respectively, and the GS $>7$ group exhibited elevated expression of HNRNPA2B1 and IGFBP3 compared with the GS $<7$ group (both $\mathrm{P}<0.05$ ) (Fig. 2G).

Consensus clustering analysis according to $\mathrm{m} 6 \mathrm{~A}$ regulators To further explore the phenotypes of m6A regulators in different prognoses and clinicopathological 
Table 2 The expression levels of 25 m6A regulators in TCGAPRAD and normal tissues

\begin{tabular}{|c|c|c|c|c|}
\hline Gene & $\begin{array}{l}\text { Normal } \\
\text { (median) }\end{array}$ & $\begin{array}{l}\text { Tumor } \\
\text { (median) }\end{array}$ & $P$ value & P symbol $^{\mathrm{a}}$ \\
\hline METTL3 & 4.304684 & 4.790198 & $2.44 \mathrm{E}-11$ & $* * *$ \\
\hline METTL14 & 3.851525 & 3.682336 & 0.03669 & $*$ \\
\hline METTL16 & 4.198874 & 3.963548 & 0.023015 & $*$ \\
\hline WTAP & 5.979951 & 5.95777 & 0.740731 & ns \\
\hline VIRMA & 4.214773 & 4.064446 & 0.255423 & ns \\
\hline ZC3H13 & 5.0224 & 4.529186 & 4.93E-08 & $* * *$ \\
\hline RBM15 & 2.901797 & 2.98326 & 0.025136 & * \\
\hline RBM15B & 5.183314 & 5.548273 & 2.44E-09 & $* * *$ \\
\hline CBLL1 & 4.733786 & 4.543428 & 0.15531 & ns \\
\hline YTHDC1 & 5.389822 & 5.331527 & 0.261206 & ns \\
\hline YTHDC2 & 3.509213 & 3.550773 & 0.057348 & ns \\
\hline YTHDF1 & 5.819509 & 6.009969 & 0.000426 & $* * *$ \\
\hline YTHDF2 & 5.58442 & 5.729154 & 0.000836 & $* * *$ \\
\hline YTHDF3 & 5.628178 & 5.457101 & 0.396418 & ns \\
\hline HNRNPC & 7.307854 & 7.455818 & 0.008174 & $* *$ \\
\hline FMR1 & 4.07059 & 3.89702 & 0.002945 & $* *$ \\
\hline LRPPRC & 5.580982 & 5.53787 & 0.397438 & ns \\
\hline HNRNPA2B1 & 7.831312 & 8.167939 & $3.34 \mathrm{E}-10$ & $* * *$ \\
\hline |GFBP1 & 0 & 0 & 0.187698 & ns \\
\hline IGFBP2 & 6.997535 & 7.466738 & $7.00 \mathrm{E}-07$ & $* * *$ \\
\hline IGFBP3 & 6.508928 & 5.863846 & 7.73E-05 & $* * *$ \\
\hline RBMX & 6.32992 & 6.408794 & 0.0266 & $*$ \\
\hline ELAVL1 & 5.323377 & 5.421104 & 0.000426 & $* * *$ \\
\hline FTO & 3.998101 & 3.576431 & $1.24 \mathrm{E}-07$ & $* * *$ \\
\hline ALKBH5 & 7.08196 & 6.930204 & 0.003862 & $* *$ \\
\hline
\end{tabular}

${ }^{a}$ ns $P>0.05 ;{ }^{*} P<0.05 ;{ }^{* *} P<0.01 ;{ }^{* * P}<0.001$

characteristics of the TCGA-PRAD cohort, we performed consensus clustering analysis to identify subgroups of 495 PARD cases according to $25 \mathrm{~m} 6 \mathrm{~A}$ regulators (m6Acluster). Relatively high consistency, a low coefficient of variation, and an appreciable increase in the area under the CDF curve were determined as the criteria of cluster number. With regard to the relative change in the area under the CDF curves for the cluster number, $k=3$ was determined to be the best category number of clusters (Fig. 3A). Subclasses were evaluated via PCA, and all three clusters were significantly distinguished, especially in clusters A and C (Fig. 3B), indicating correct prediction of the m6Acluster (these clusters are shown as "clusters 1-3 or A-C"). Next, we compared different clinicopathological characteristics of PRAD among the clusters, and the results showed relatively higher pathological $\mathrm{N}(\mathrm{pN})$ stage, GS 8-10, and biochemical recurrence rates in cluster A (Fig. 3C). The different KEGG pathways between clusters $\mathrm{A}$ and $\mathrm{C}$ were analyzed through GSVA (Fig. 3D). Cancer- and apoptosis-related pathways were significantly dysregulated between clusters A and C. We also analyzed changes in immune cell infiltration in different m6Aclusters and found 12 of 23 subpopulations of immune cells to be differentially expressed in the three clusters (Fig. 3E and Table 6). Kaplan-Meier survival curves of RFS based on the m6Aclusters demonstrated no significant differences among the groups $(\mathrm{P}=0.475)$ (Fig. 3F).

\section{Consensus clustering analysis based on DEGs of m6Aclusters}

DEGs ( $P$ value of Bayes test $<0.001$ ) were analyzed in every pairwise comparison according to the three clusters based on m6A regulators, and the 74 intersecting genes were identified via a Venn diagram (Fig. 4A). We then analyzed the biological functions of these 74 genes, which were categorized into GO terms of biological process (BP), cell component (CC), and molecular function (MF). Under the stringent threshold of P-adjust $<0.05$, only 1 specific CC (proton-transporting V-type ATPase, V0 domain) was enriched (Additional file 2: Fig. S1AB). Additionally, KEGG signaling pathway analysis of 74 genes indicated significant enrichment in the oxidative phosphorylation KEGG pathway (Additional file 2: Fig. S1C-D).

Univariate Cox regression analysis was also performed on the 74 intersecting genes, and 6 (BAIAP2, TEX264, MMAB, JAGN1, TIMM8AP1, and IMP3) related to PCa recurrence were selected $(\mathrm{P}<0.05)$ (Fig. 4B). The characteristics of the 6 or 5 DEGs (TIMM8AP1 was excluded because of being a processed pseudogene and a lack of copy number data) regarding CNV alterations, DEGs, and mutation frequency were analyzed in $\mathrm{PCa}$ compared with normal samples (Additional file 3: Fig. S2A-D and Additional file 1: Table S2). We found that the levels of 4 (BAIAP2, IMP3, JAGN1, MMAB) of the 6 genes were significantly upregulated in $\mathrm{PCa}$ tissues. Interestingly, JAGN1 exhibited the highest degree of copy number loss (5.18\%), but its expression was higher in PCa tissues than in normal prostate tissues $(\mathrm{P}<0.01)$. When assessing the expression of the 6 genes with respect to the clinicopathological characteristics GS, pT, and TP53, we found that most were downregulated in advanced stages (GS > 7, pT3, and TP53 mutation). Survival analysis also indicated that low expression of these 6 genes was significantly associated with poor RFS (Additional file 4: Fig. S3A-D and Additional file 1: Table S3-S5). The risk factors and relationships of the 6 genes are summarized in the prognosis network illustrated in Additional file 4: Fig. S3E.

Consensus clustering analysis was performed for these 6 genes (geneCluster), and $k=3$ was determined to result in the best classification with respect to the delta area results (cluster 1-3 or A-C) (Fig. 4C). PCA verified the 


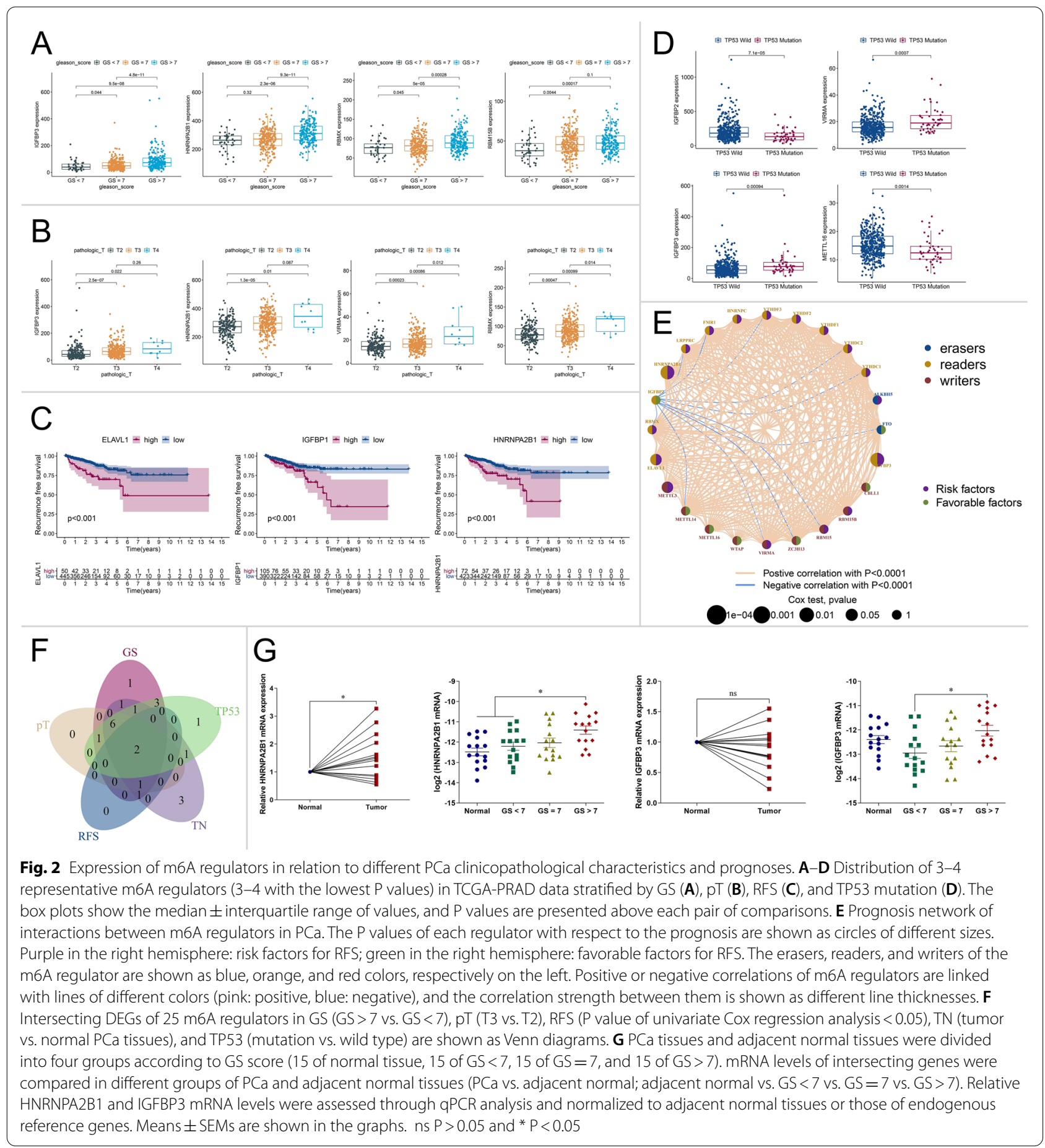

significance of the three subgroups (Additional file 5: Fig. S4A).

A heatmap of clinicopathological features in the three geneClusters indicated that $\mathrm{pT}$ stage, N stage, GS, and biological recurrence were significantly higher in geneCluster A than in the other clusters (Fig. 4D). KaplanMeier survival analysis revealed significant differences between the three m6Aclusters, among which geneCluster A had the poorest RFS ( $\mathrm{P}=0.012)$ (Fig. 4E). Next, we analyzed the expression of $\mathrm{m} 6 \mathrm{~A}$ regulators in different geneClusters and found that 21 of $25 \mathrm{~m} 6 \mathrm{~A}$ regulators were differentially expressed among them (Fig. $4 \mathrm{~F}$ and Table 7). Moreover, 16 of 23 subpopulations of immune cells were differentially expressed among the three 
Table 3 Expression levels of m6A regulators in TCGA-PRAD data stratified by GS

\begin{tabular}{|c|c|c|c|c|c|}
\hline Gene & $\mathrm{GS}<7$ (median) & $\mathrm{GS}=7$ (median) & GS > 7 (median) & $\mathrm{P}(\mathrm{GS}<7$ vs. GS $>7)$ & P symbol $^{\mathrm{a}}$ \\
\hline METTL3 & 25.86643 & 26.03757 & 28.77871 & 0.012126 & * \\
\hline METTL14 & 11.3965 & 12.04075 & 11.73067 & 0.587863 & ns \\
\hline METTL16 & 14.60147 & 15.50221 & 14.0438 & 0.44366 & ns \\
\hline WTAP & 62.4395 & 63.1373 & 57.53999 & 0.143969 & ns \\
\hline VIRMA & 14.15936 & 15.21605 & 16.58136 & 0.000484 & $* * *$ \\
\hline ZC3H13 & 23.46752 & 22.64276 & 21.06241 & 0.589439 & ns \\
\hline RBM15 & 6.503603 & 6.872581 & 7.042117 & 0.030353 & * \\
\hline RBM15B & 37.72488 & 45.43605 & 47.3531 & 0.00017 & $* * *$ \\
\hline CBLL1 & 23.24536 & 22.96488 & 21.80928 & 0.93803 & ns \\
\hline YTHDC1 & 38.39455 & 38.13983 & 40.96145 & 0.012687 & * \\
\hline YTHDC2 & 10.07219 & 10.39407 & 11.02557 & 0.050573 & ns \\
\hline YTHDF1 & 56.35877 & 63.87374 & 65.1596 & 0.000336 & $* * *$ \\
\hline YTHDF2 & 47.1231 & 53.60412 & 52.07434 & 0.007882 & $* *$ \\
\hline YTHDF3 & 38.58748 & 43.74472 & 44.60192 & 0.009576 & $* *$ \\
\hline HNRNPC & 148.352 & 171.741 & 180.9566 & 0.000415 & $* * *$ \\
\hline FMR1 & 12.6015 & 13.62071 & 14.75719 & 0.000992 & $* * *$ \\
\hline LRPPRC & 41.37827 & 44.88092 & 47.23535 & 0.009325 & $* *$ \\
\hline HNRNPA2B1 & 263.0709 & 271.8228 & 310.4768 & $2.31 \mathrm{E}-06$ & $* * *$ \\
\hline IGFBP1 & 0 & 0 & 0.013463 & 0.024217 & $*$ \\
\hline IGFBP2 & 187.1589 & 182.7438 & 161.6246 & 0.026865 & $*$ \\
\hline IGFBP3 & 39.02542 & 48.8547 & 72.32879 & $9.45 \mathrm{E}-08$ & $* * *$ \\
\hline $\mathrm{RBMX}$ & 76.6101 & 81.48018 & 88.62738 & 4.93E-05 & $* * *$ \\
\hline ELAVL1 & 39.58125 & 41.91052 & 42.85327 & 0.0035 & $* *$ \\
\hline FTO & 11.43039 & 11.10629 & 10.74576 & 0.705953 & ns \\
\hline ALKBH5 & 120.1901 & 121.221 & 120.7401 & 0.878236 & ns \\
\hline
\end{tabular}

a ns $\mathrm{P}>0.05 ;{ }^{*} \mathrm{P}<0.05 ;{ }^{*} \mathrm{P}<0.01 ;{ }^{* *} \mathrm{P}<0.001$

clusters (Additional file 5: Fig. S4B and Additional file 1: Table S6). In the GSVA of different KEGG pathways, a small cell lung cancer-related pathway was dysregulated between geneClusters $\mathrm{A}$ and $\mathrm{C}$ (Additional file 5: Fig. S4C).

We also analyzed DEGs of the 6 genes regarding GS, pT, RFS, TN, and TP53, as illustrated in Fig. 2G. The intersecting genes MMAB and BAIAP2 were identified via a Venn diagram (Fig. 4G), and both were highly expressed in $\mathrm{PCa}$ tissues compared with normal tissue but were low with respect to the four parameters, indicating advanced stage. This suggests opposite expression patterns of MMAB and PAIAP2 in PCa occurrence and progression. The mRNA expression level of $M M A B$ was analyzed through qPCR using 45 PCa tissues and 15 adjacent normal prostate tissues, as mentioned above. It was upregulated in $60.0 \%(9 / 15, \mathrm{P}<0.05)$ of $\mathrm{PCa}$ tissues compared with adjacent normal tissues, with the GS $>7$ group exhibiting reduced expression compared with the $\mathrm{GS}<7$ group $(\mathrm{P}<0.05)$ (Fig. $4 \mathrm{H})$. The differential expression of MMAB in $\mathrm{PCa}$ occurrence and progression was histologically verified.

\section{Low m6Ascore based on the 6 genes was associated with poor prognosis in RFS}

The clustering analysis above was based on the population of the patient and could not be used to quantitatively assess the patterns of $\mathrm{m} 6 \mathrm{~A}$ regulators. Considering the complexity of m6A regulators and individual heterogeneity, the m6Ascore was calculated through PCA of the 6 gene levels in TCGA-PARD, and statistical analysis was performed to examine the relationship between m6Ascore and geneCluster/m6Acluster. The results revealed a significant difference in both clusters: geneCluster A had the lowest m6Ascore, and geneCluster $\mathrm{C}$ had the highest (Fig. 5A). PRAD patients were divided into two groups based on the optimum threshold segmentation of m6Ascore in RFS analysis, and according to Kaplan-Meier survival analysis, poor RFS was associated with the low m6Ascore group (Fig. 5B). The alluvial diagram shows the attribute changes in m6Acluster, geneCluster, m6Ascore, and recurrence status (fustat) (Fig. 5C). All patients in the low m6Ascore group were classified into geneCluster A, which was relevant to the worse RFS outcome (recurrence rate: $27.6 \%$ ). The correlation of m6Ascore 
Table 4 Expression levels of m6A regulators in TCGA-PRAD data stratified by pT

\begin{tabular}{|c|c|c|c|c|c|}
\hline Gene & $\mathrm{pT}=2$ (median) & $\mathrm{pT}=3$ (median) & $\mathrm{pT}=4$ (median) & $\mathrm{P}(\mathrm{pT}=2$ vs. $\mathrm{pT}=3)$ & P symbol $^{a}$ \\
\hline METTL3 & 27.06274 & 26.48476 & 26.30134 & 0.365576 & ns \\
\hline METTL14 & 11.67474 & 11.8227 & 13.97603 & 0.853312 & ns \\
\hline METTL16 & 15.01602 & 14.43229 & 17.49281 & 0.229372 & ns \\
\hline WTAP & 62.01344 & 59.79612 & 53.04483 & 0.06393 & ns \\
\hline VIRMA & 14.40579 & 16.51078 & 22.99954 & 0.000227 & $* * *$ \\
\hline ZC3H13 & 23.14228 & 21.01365 & 22.76672 & 0.416107 & ns \\
\hline RBM15 & 6.749674 & 6.883484 & 8.615314 & 0.274807 & ns \\
\hline RBM15B & 44.14219 & 46.68428 & 55.24486 & 0.0486 & * \\
\hline CBLL1 & 23.00583 & 21.84154 & 30.68415 & 0.360921 & ns \\
\hline YTHDC1 & 38.16906 & 39.80338 & 44.09208 & 0.062198 & ns \\
\hline YTHDC2 & 10.47902 & 10.7769 & 17.22446 & 0.439427 & ns \\
\hline YTHDF1 & 61.14875 & 64.5393 & 87.48373 & 0.011815 & * \\
\hline YTHDF2 & 51.36317 & 52.29989 & 68.72696 & 0.336839 & ns \\
\hline YTHDF3 & 41.22466 & 44.1152 & 62.55585 & 0.148109 & ns \\
\hline HNRNPC & 167.5473 & 179.2224 & 204.646 & 0.001177 & $* *$ \\
\hline FMR1 & 13.15182 & 14.1732 & 20.4288 & 0.001644 & $* *$ \\
\hline LRPPRC & 44.08828 & 46.08805 & 60.92826 & 0.249137 & ns \\
\hline HNRNPA2B1 & 271.8575 & 296.6347 & 344.7956 & 1.31E-05 & $* * *$ \\
\hline IGFBP1 & 0 & 0 & 0.038231 & 0.100044 & ns \\
\hline IGFBP2 & 187.1589 & 168.3542 & 152.7416 & 0.037715 & * \\
\hline IGFBP3 & 42.2307 & 64.22177 & 83.39194 & $2.48 \mathrm{E}-07$ & $* * *$ \\
\hline RBMX & 78.0526 & 86.94696 & 119.5574 & 0.000471 & $* * *$ \\
\hline ELAVL1 & 40.91439 & 42.45284 & 49.47014 & 0.002996 & $* *$ \\
\hline FTO & 10.76108 & 10.90775 & 13.05522 & 0.967256 & ns \\
\hline ALKBH5 & 121.6235 & 120.1459 & 132.7814 & 0.579113 & ns \\
\hline
\end{tabular}

${ }^{a}$ ns $\mathrm{P}>0.05 ;{ }^{*} \mathrm{P}<0.05 ;{ }^{* *} \mathrm{P}<0.01 ;{ }^{* * * P}<0.001$

and immune-associated genes was evaluated, resulting in 16 of 23 immune-related cell infiltrates being found to be negatively related to m6Ascore (Fig. 5D). This suggests the negative regulation of the immune reaction during m6Ascore elevation. A previous study showed that low immune infiltration was potentially related to prolonged survival [26]. Consistent with our results, the high m6Ascore group had a tendency toward favorable prognosis in terms of RFS (Fig. 5B).

\section{Characteristics of m6Ascore status for TCGA-PRAD tumor mutation and subtypes}

A study suggested that the TMB score was positively associated with tumor-related oncogenic mutations, which led to tumor progression but was easier to detect by immune cells, making them more likely to be sensitive to immunotherapy $[27,28]$. As shown in Fig. 5B, TCGA-RRAD patients were divided into two groups (58 in the low m6Ascore group and 437 in the high m6Ascore group), and the association of m6Ascore and TMB was assessed. The results showed no significant difference in TMB scores in the m6Ascore groups $(\mathrm{P}=0.13)$
(Fig. 6A, left). Spearman correlation analysis also showed no significant correlation between the expression levels of m6Ascore and TMB $(\mathrm{P}>0.05)$ (Fig. 6A, right). This is discordant with other study in gastric cancer, which showed that m6Ascore and TMB exhibited a significant negative correlation [19]. The PRAD patients were then divided into two groups based on the optimum threshold segmentation of TMB value in RFS analysis (242 in the low TMB group and 231 in the high TMB group). In Kaplan-Meier analysis, a tendency toward poor RFS was found in the high TMB group compared with the low TMB group, but the difference was not significant $(\mathrm{P}=0.051)$ (Fig. 6B, left). This suggests that the higher malignancy of $\mathrm{PCa}$ patients potentially correlated with the high TMB score group. When analyzing m6A and TMB together, we found a great RFS advantage for the combination of low TMB with high m6Ascore (Fig. 6B, right). Consistent with our previous result, the low TMB and high m6Ascore groups had better prognoses in terms of RFS (Fig. 5B and Fig. 6B, left).

The distribution differences of somatic mutations were analyzed between m6Ascore groups, and the results 
Table 5 Expression levels of m6A regulators in TCGA-PRAD data stratified by TP53 mutation

\begin{tabular}{|c|c|c|c|c|}
\hline Gene & Wild type (median) & Mutation (median) & $P$ value (wild vs. mut) & P symbol $^{a}$ \\
\hline METTL3 & 26.66187 & 28.77871 & 0.155657 & ns \\
\hline METTL14 & 11.7543 & 11.92501 & 0.210393 & ns \\
\hline METTL16 & 14.83022 & 12.4188 & 0.001348 & $* *$ \\
\hline WTAP & 61.08309 & 59.07833 & 0.694618 & ns \\
\hline VIRMA & 15.52701 & 18.9505 & 0.000697 & $* * *$ \\
\hline ZC3H13 & 20.69471 & 24.60509 & 0.071727 & ns \\
\hline RBM15 & 6.887572 & 6.931969 & 0.381289 & ns \\
\hline RBM15B & 46.10196 & 52.61076 & 0.008893 & $* *$ \\
\hline CBLL1 & 21.93925 & 24.83281 & 0.144508 & ns \\
\hline YTHDC1 & 38.77673 & 44.14293 & 0.01052 & * \\
\hline YTHDC2 & 10.51239 & 12.71358 & 0.008028 & $* *$ \\
\hline YTHDF1 & 62.76196 & 66.50237 & 0.045122 & * \\
\hline YTHDF2 & 51.76176 & 60.97605 & 0.011735 & * \\
\hline YTHDF3 & 42.92899 & 48.0619 & 0.037743 & * \\
\hline HNRNPC & 175.4147 & 186.623 & 0.013856 & * \\
\hline FMR1 & 13.67919 & 15.39546 & 0.028109 & * \\
\hline LRPPRC & 45.50271 & 55.43345 & 0.04845 & * \\
\hline HNRNPA2B1 & 285.6907 & 320.1461 & 0.005563 & $* *$ \\
\hline IGFBP1 & 0 & 0 & 0.328748 & ns \\
\hline IGFBP2 & 182.2817 & 130.7453 & $7.05 \mathrm{E}-05$ & $* * *$ \\
\hline IGFBP3 & 54.82908 & 76.41379 & 0.000933 & $* * *$ \\
\hline RBMX & 82.82156 & 93.84054 & 0.009741 & $* *$ \\
\hline ELAVL1 & 42.00761 & 42.3959 & 0.435743 & ns \\
\hline FTO & 10.87488 & 9.600595 & 0.738732 & ns \\
\hline ALKBH5 & 119.9799 & 121.8514 & 0.551667 & ns \\
\hline
\end{tabular}

${ }^{a}$ ns $P>0.05 ;{ }^{*} P<0.05 ; *{ }^{*} P<0.01 ; * * P<0.001$

indicated a more extensive mutation frequency of TP53 $(18 \%$ vs. $8 \%)$ but a reduced mutation frequency of SPOP ( $2 \%$ vs. $12 \%$ ) in the low m6Ascore group (Fig. 6C).

For the relationship between m6Ascore and fustat, the low m6Ascore group experienced a higher rate of recurrence (Fig. 6D left), which was consistent with the RFS analysis in Fig. 5B. Biochemical recurrence was also associated with lower m6Ascore (Fig. 6D, right). We then analyzed prognosis in the m6Ascore groups with regard to different clinicopathological features through RFS analysis (Fig. 6E and Additional file 6: Fig. S5A-B). The low m6Ascore group was associated with poor RFS in GS $8-10$ and $\mathrm{pT} 3$ stages $(\mathrm{P}<0.05)$ (Fig. 6E).

The expression of PD-L1 was detected to elucidate a potential response to immunotherapy, and the low m6Ascore group showed relatively high levels of expression $(P=0.023)$ (Fig. 6F). Finally, we further predicted the value of the risk score for immune checkpoint blockade (ICB). The IPS results of TCGA-PRAD were downloaded from the TCIA website. PD1 and CTLA4 were enrolled for IPS analysis and further classified into four parts: ips_ctla4_neg_pd1_neg (CTLA4 negative response and
PD1 negative response), ips_ctla4_neg_pd1_pos (CTLA4 negative response and PD1 positive response), ips_ctla4 pos_pd1_neg, and ips_ctla4_pos_pd1_pos (Fig. 6G, Additional file 6: Fig. S5C). The average IPS in the comparison of the low and high m6Ascore groups showed no significant difference in the four parts of the negative or positive response of PD1 and CTLA4 (Fig. 6G, Additional file 6: Fig. $\mathrm{S} 5 \mathrm{C}$ ). These results indicate that m6Ascore may lack efficacy in the risk score model for predicting the response to PD1 and CTLA4 therapy.

\section{Discussion}

In this study, we comprehensively evaluated the m6A modification patterns in TCGA-PRAD and found 6 DEGs (BAIAP2, TEX264, MMAB, JAGN1, TIMM8AP1, and IMP3) based on m6Aclusters. A model of optimal clusters was established, and m6Ascore analysis was performed based on the above 6 genes.

In general, elucidating the molecular mechanism of PCa progression remains crucial. It has been reported that epigenetic reprogramming is key for $\mathrm{PCa}$ progression $[29,30]$ and that inhibition of the epigenetic 
A

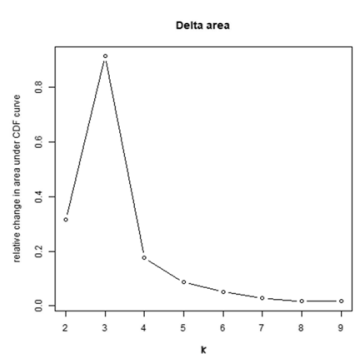

C

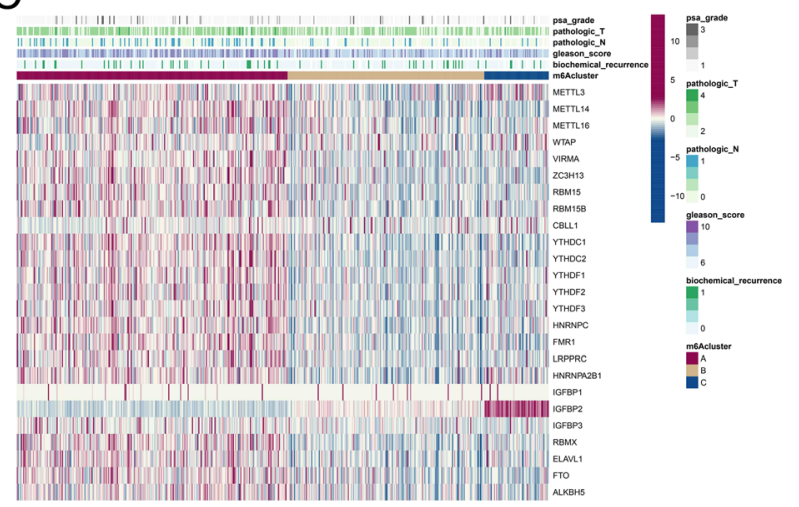

$\mathrm{E}$
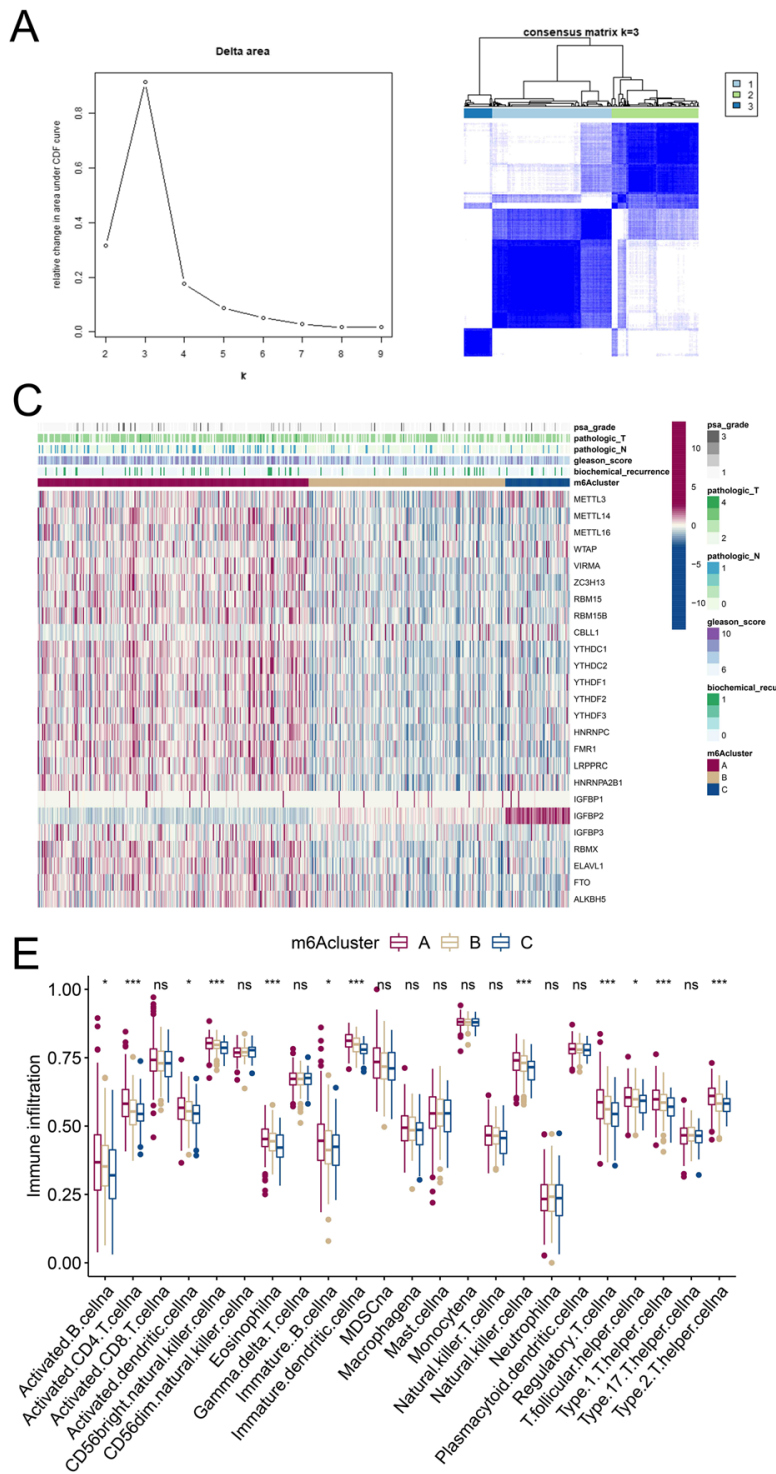

B

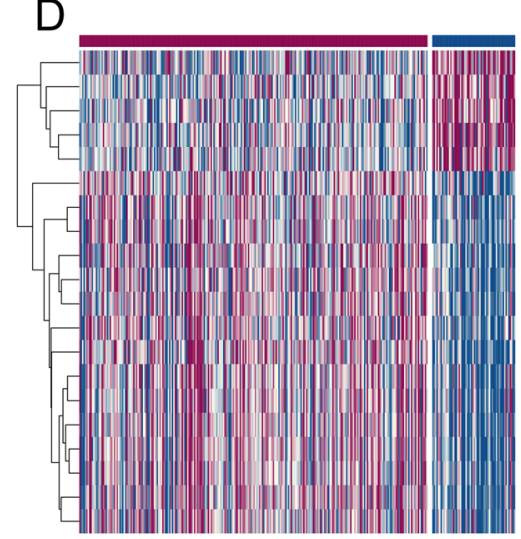

m6Acluster

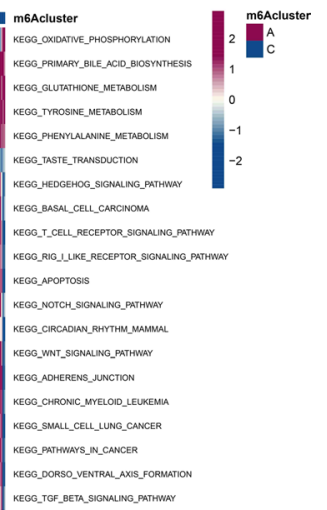

$\mathrm{F}$

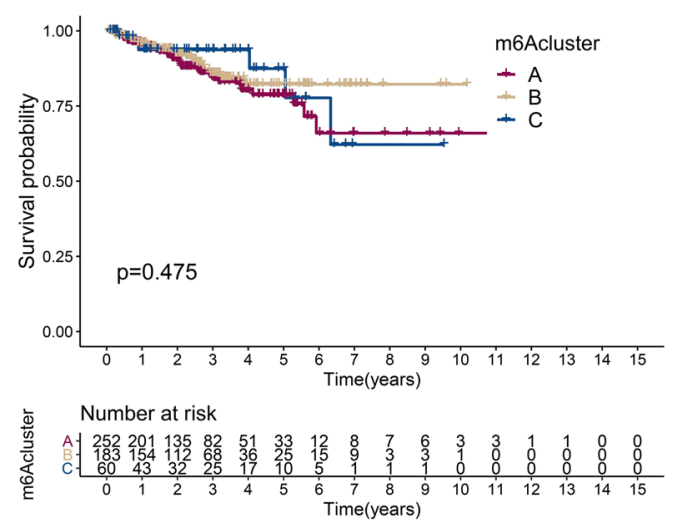

Fig. 3 Consensus clustering analysis based on m6A regulators. A (left) Relative change in area under the CDF curve from $k=2$ to 9. (right) Color-coded heatmap of the consensus matrix for $\mathrm{k}=3$. Color gradients represent values from $0-1$ (white: 0 , dark blue: 1). B PCA of the transcriptome profiles of three m6Acluster patterns, showing a marked difference in the transcriptome between different m6Aclusters. Different dots of red, orange, and blue in the scatter diagram represent m6Aclusters A to C. C The heatmap shows unsupervised clustering of $25 \mathrm{~m} 6 \mathrm{~A}$ regulators in TCGA-PRAD. PSA grade, pT, pN, GS, and biochemical recurrence were used for patient annotation. Red represents high expression, and blue represents low expression. D Heatmap of KEGG enrichment analysis showing the activation states of biological pathways in distinct m6Aclusters. Red represents activation, and blue represents inhibition. E Abundance of each infiltrating immune cell among the three m6Aclusters is shown in the boxplot. The median \pm interquartile range of values is shown in the graph. ns $P>0.05 ;{ }^{*} P<0.05 ;{ }^{* * *} P<0.001$. F Survival analysis for RFS among three m6Aclusters based on 495 TCGA-PRAD patients. Kaplan-Meier curves and log-rank $P$ values are shown in the graph, and the numbers at risk are shown at the bottom

regulator EZH2 might effectively overcome ADT resistance [2]. Our previous study found that epigenetically activating AR/NDRG1 signaling through histone methylation and DNA methylation significantly suppresses CRPC progression [20, 21].
Nevertheless, the effect of mRNA posttranscriptional modification in $\mathrm{PCa}$ is still unclear. m6A RNA methylation may be among the most extensive RNA modifications [31-33]. Yabing Chen et al. highlighted that total RNA m6A modification levels are significantly increased 
Table 6 Abundance of each infiltrating immune cell among the three m6Aclusters

\begin{tabular}{|c|c|c|c|c|c|}
\hline Immune cell infiltration & Group A (median) & Group B (median) & Group C (median) & P value & P symbol $^{\mathrm{a}}$ \\
\hline Activated.B.cellna & 0.367609 & 0.35241 & 0.320044 & 0.035299 & * \\
\hline Activated.CD4.T.cellna & 0.581698 & 0.553239 & 0.544626 & $2.04 \mathrm{E}-07$ & $* * *$ \\
\hline Activated.CD8.T.cellna & 0.741994 & 0.729711 & 0.729734 & 0.119033 & ns \\
\hline Activated.dendritic.cellna & 0.566906 & 0.55417 & 0.545927 & 0.014999 & * \\
\hline CD56bright.natural.killer.cellna & 0.803422 & 0.796865 & 0.786135 & 0.000248 & $* * *$ \\
\hline CD56dim.natural.killer.cellna & 0.768694 & 0.770296 & 0.777682 & 0.468408 & ns \\
\hline Eosinophilna & 0.452763 & 0.44455 & 0.421455 & 0.000297 & $* * *$ \\
\hline Gamma.delta.T.cellna & 0.672574 & 0.672239 & 0.675783 & 0.999306 & ns \\
\hline Immature..B.cellna & 0.446315 & 0.41228 & 0.424357 & 0.01113 & * \\
\hline Immature.dendritic.cellna & 0.812085 & 0.79882 & 0.779721 & 1.15E-10 & $* * *$ \\
\hline MDSCna & 0.734421 & 0.71748 & 0.710971 & 0.169041 & ns \\
\hline Macrophagena & 0.493925 & 0.484737 & 0.485948 & 0.440833 & ns \\
\hline Mast.cellna & 0.546259 & 0.546121 & 0.546628 & 0.844207 & ns \\
\hline Monocytena & 0.880654 & 0.87941 & 0.879403 & 0.913355 & ns \\
\hline Natural.killer.T.cellna & 0.466253 & 0.464513 & 0.456207 & 0.069941 & ns \\
\hline Natural.killer.cellna & 0.74012 & 0.730841 & 0.715254 & 1.39E-05 & $* * *$ \\
\hline Neutrophilna & 0.233375 & 0.241803 & 0.236327 & 0.692117 & ns \\
\hline Plasmacytoid.dendritic.cellna & 0.781692 & 0.779419 & 0.777867 & 0.247269 & ns \\
\hline Regulatory.T.cellna & 0.586997 & 0.561565 & 0.544363 & 0.000173 & $* * *$ \\
\hline T.follicular.helper.cellna & 0.604853 & 0.598244 & 0.592525 & 0.029061 & * \\
\hline Type.1.T.helper.cellna & 0.597887 & 0.585768 & 0.570837 & 0.000293 & $* * *$ \\
\hline Type.17.T.helper.cellna & 0.466109 & 0.466299 & 0.464527 & 0.584038 & ns \\
\hline Type.2.T.helper.cellna & 0.61026 & 0.582465 & 0.582235 & 3.01E-09 & $* * *$ \\
\hline
\end{tabular}

a ns $\mathrm{P}>0.05 ;{ }^{*} \mathrm{P}<0.05 ;{ }^{* *} \mathrm{P}<0.01 ;{ }^{* * *} \mathrm{P}<0.001$

in PCa tissues due to upregulation of METTL3 [34]. In addition, knockdown of METTL3 significantly reduces PCa cell migration and invasion. YTHDF2, a reader of m6A modification, synergistically induces PCa progression with METTL3 by regulating AKT phosphorylation [15]. However, low levels of METTL3 were also found to lead to advanced metastatic PCa that is resistant to androgen receptor antagonists [35]. These controversial results reveal the complexity of $\mathrm{m} 6 \mathrm{~A}$ regulators and individual heterogeneity of $\mathrm{m} 6 \mathrm{~A}$ regulators in $\mathrm{PCa}$, and various $\mathrm{m} 6 \mathrm{~A}$ regulators may form a complex network structure and interact with each other to affect $\mathrm{PCa}$ progression.

In this study, we examined three representative groups of m6A regulators: 14 "readers", 9 "writers", and 2 "erasers". Bioinformatic analysis was then performed to comprehensively investigate the association between m6A modification patterns and PCa clinicopathological characteristics and prognosis.

Compared with normal prostate tissues in TCGA database, METTL3 was significantly highly expressed in PCa, and negligible $\mathrm{CNV}$ and mutation rate were found in this analysis. ZC3H13 exhibited the highest degree of copy number loss $(28.49 \%)$ and highest mutation rate $(4 / 484)$ among all $25 \mathrm{~m} 6 \mathrm{~A}$ regulators. The mRNA of ZC3H13 was also expressed at low levels in the TN comparison $(\mathrm{P}<0.001)$. Therefore, a potential mechanism including ZC3H13 mutation may exist in PCa progression. Insufficient study has been conducted to elucidate this point, which warrants further verification.

In survival and Cox regression analyses, we evaluated RFS instead of overall survival (OS) because only 10 of 493 TCGA-PARD patients died, making it difficult to obtain statistically significant results by assessing OS. In our RFS analysis, "biochemical_recurrence" and "days_ to_first_biochemical_recurrence" data did not coincide, and we ultimately used the "days_to_first_biochemical_recurrence" data to confirm "recurrence status" and "recurrence follow-up time".

The landscape of m6A variation in PCa was recently reported [26, 36-40], although no scholars have analyzed sufficient $\mathrm{m} 6 \mathrm{~A}$ regulators and verified them using clinical PCa tissues. To overcome these shortcomings, we explored all 25 widely-acknowledged $\mathrm{m} 6 \mathrm{~A}$ regulators and assessed relevant associations of various clinicopathological characteristics, such as TN, GS, pT, TP53 mutation, and survival analysis of RFS using TCGA-PRAD data. Additionally, we analyzed DEGs of m6A regulators 


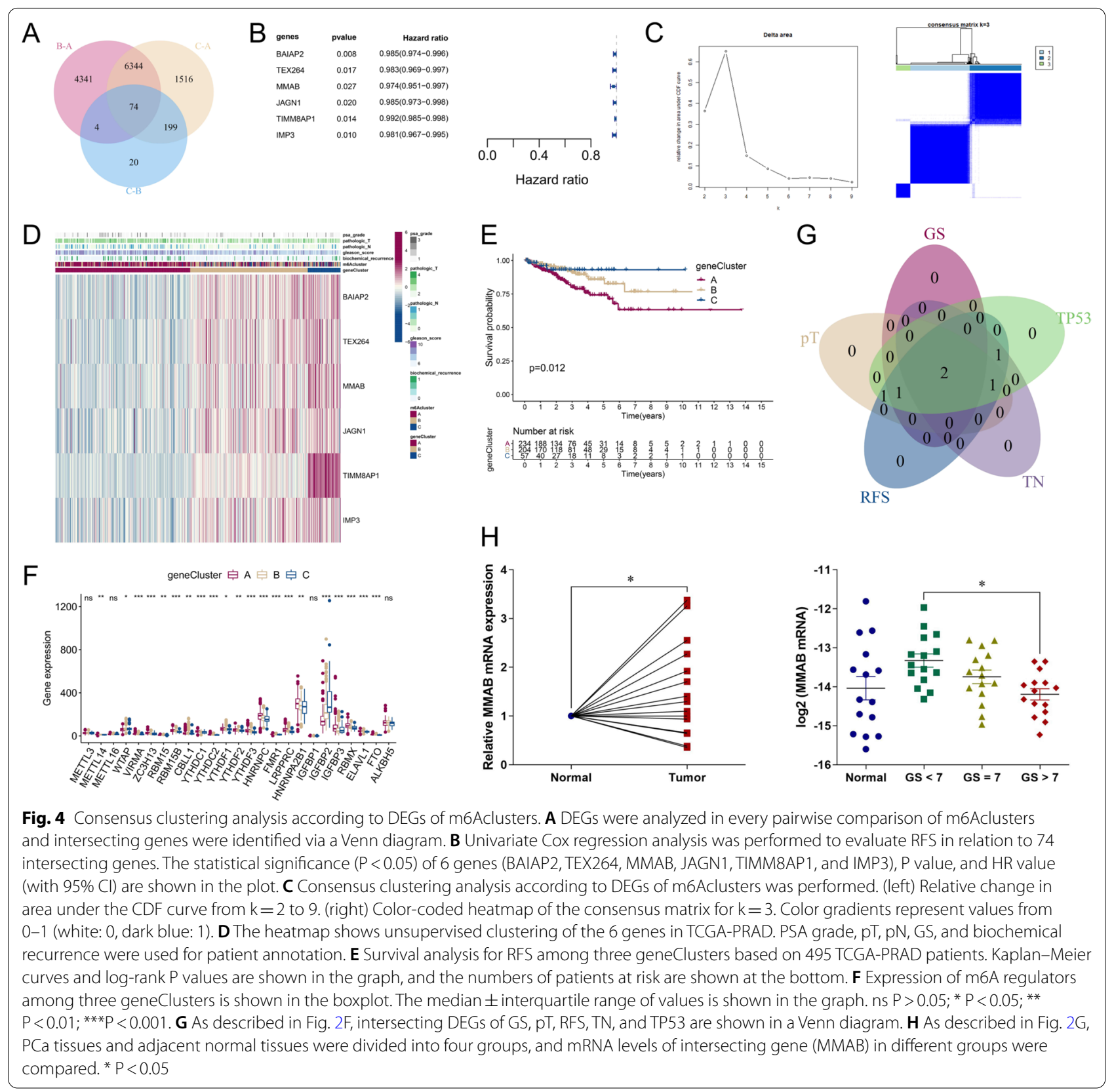

in relation to the five factors, and the intersecting genes (HNRNPA2B1 and IGFBP3) were verified with clinical $\mathrm{PCa}$ tissues. The level of HNRNPA2B1 was higher in PCa tissues than in normal prostate tissues and was highly correlated with the other four advanced factors. However, the level of IGFBP3 was lower in PCa tissues but higher in the presence of the above advanced-stage indicators. This suggests that independent molecular mechanisms and expression patterns exist in PCa occurrence and progression. Consistent with the bioinformatic analysis, the mRNA levels of HNRNPA2B1 and IGFBP3 were elevated in PCa tissue of the high GS group $(G S>7)$ compared with the low GS group $(\mathrm{GS}<7)$. In the TN comparison, the expression of HNRNPA2B1 was higher in PCa, but no significant difference in the level of IGFBP3 expression was found.

It has been reported that upregulation of HNRNPA2B1 by PCAT6 promotes PCa progression and neuroendocrine differentiation [41]. HNRNPA2B1 may also be an independent prognostic factor and contribute to cancer progression [42]. Coexpression network analysis using clinical data from the GSE70768 dataset as well 
Table 7 Expression of m6A regulators among three geneClusters

\begin{tabular}{|c|c|c|c|c|c|}
\hline Gene & Group A (median) & Group B (median) & Group C (median) & $P$ value & P symbol $^{a}$ \\
\hline METTL3 & 25.43239 & 28.121 & 27.4013 & 0.067214 & ns \\
\hline METTL14 & 12.49848 & 11.61563 & 10.65721 & 0.002169 & $* *$ \\
\hline METTL16 & 15.11261 & 14.27289 & 14.50079 & 0.198853 & ns \\
\hline WTAP & 59.59774 & 61.67926 & 63.11492 & 0.011085 & * \\
\hline VIRMA & 17.50783 & 14.85196 & 13.20034 & 5.68E-09 & $* * *$ \\
\hline ZC3H13 & 24.27743 & 19.83391 & 16.76071 & $9.62 \mathrm{E}-06$ & $* * *$ \\
\hline RBM15 & 7.196895 & 6.848082 & 6.450624 & 0.001906 & $* *$ \\
\hline RBM15B & 50.11591 & 45.15223 & 37.2346 & 1.84E-09 & $* * *$ \\
\hline CBLL1 & 21.41409 & 24.31778 & 19.51026 & 0.001104 & $* *$ \\
\hline YTHDC1 & 41.63361 & 37.73905 & 36.59234 & $7.15 \mathrm{E}-07$ & $* * *$ \\
\hline YTHDC2 & 11.26867 & 10.11635 & 8.712406 & $1.55 \mathrm{E}-06$ & $* * *$ \\
\hline YTHDF1 & 65.1071 & 63.04105 & 58.22785 & 0.027657 & * \\
\hline YTHDF2 & 55.0904 & 51.34166 & 48.46479 & 0.00317 & $* *$ \\
\hline YTHDF3 & 47.39185 & 42.42069 & 33.32456 & 6.17E-08 & $* * *$ \\
\hline HNRNPC & 187.0431 & 166.5035 & 152.1311 & $2.12 \mathrm{E}-08$ & $* * *$ \\
\hline FMR1 & 15.0888 & 13.23247 & 12.25827 & 1.79E-07 & $* * *$ \\
\hline LRPPRC & 53.01479 & 43.53207 & 37.2318 & 1.07E-08 & $* * *$ \\
\hline HNRNPA2B1 & 299.7436 & 280.6586 & 271.21 & 0.001427 & $* *$ \\
\hline IGFBP1 & 0 & 0 & 0 & 0.057215 & ns \\
\hline IGFBP2 & 133.3923 & 214.2155 & 266.8425 & $2.11 \mathrm{E}-25$ & $* * *$ \\
\hline IGFBP3 & 66.44944 & 43.65073 & 48.12219 & 4.87E-08 & $* * *$ \\
\hline RBMX & 92.67909 & 79.35541 & 72.55706 & $1.94 \mathrm{E}-12$ & $* * *$ \\
\hline ELAVL1 & 44.32754 & 41.28624 & 39.84319 & 0.000294 & $* * *$ \\
\hline FTO & 11.70362 & 10.25875 & 7.217273 & 7.92E-09 & $* * *$ \\
\hline ALKBH5 & 122.1352 & 121.0671 & 118.568 & 0.116104 & ns \\
\hline
\end{tabular}

a ns $\mathrm{P}>0.05 ;{ }^{*} \mathrm{P}<0.05 ;{ }^{* *} \mathrm{P}<0.01 ;{ }^{* *} \mathrm{P}<0.001$

as quantitative proteomic mass spectrometry profiling and gene enrichment analysis using $\mathrm{LNCaP}$ and PC3 cell lines suggest that HNRNPA2B1 is associated with $\mathrm{PCa}$ progression and prognosis [43, 44]. The level of IGFBP3 in $\mathrm{PCa}$ is controversial, with one meta-analysis indicating that the CC genotype of the IGFBP3-202A/C polymorphism is associated with an increased risk of $\mathrm{PCa}$ [45-47]. Overall, tissue verification and previous studies support a certain level of accuracy of our bioinformatic analysis using TCGA-PRAD data.

To analyze the overall effects of m6A regulators in $\mathrm{PCa}$, we performed consensus clustering analysis and divided the TCGA-PRAD cohort into three subgroups based on $25 \mathrm{~m} 6 \mathrm{~A}$ regulators. As no significant difference in the three clusters for RFS was observed, this cluster analysis with $\mathrm{m} 6 \mathrm{~A}$ regulators may not be suitable for inclusion in a prognostic risk prediction model.

As epigenetic regulators, posttranscriptional modification of target genes is the primary function of m6A regulators. To analyze the downstream genes of m6A regulators, we focused on the DEGs of the m6Acluster and screened six risk genes (BAIAP2, TEX264,
MMAB, JAGN1, TIMM8AP1, and IMP3) through univariate Cox regression analysis. Only IMP3 has previously been reported in PCa studies, and it appears to be increased in $\mathrm{PCa}$ tissues and associated with higher GS, PCa metastasis, and PCa-specific survival [48-50]. Regardless, according to our bioinformatic analysis of TCGA-PRAD, the expression of IMP3 was decreased in three groups related to advanced PCa $(\mathrm{HR}(\mathrm{RFS})<1$, TP53 mutation $<$ wild type, $\mathrm{pT} 3<\mathrm{pT} 2$, and tumor $>$ normal). This finding indicates discordance between TCGAPRAD data and previous results, which warrants further confirmation.

Consensus clustering analysis was then performed based on the 6 genes to construct a risk prediction model, and TCGA-PARD patients were divided into three subgroups. In these clusters, the clinicopathological parameters of PSA grade 3 (grade 1: $>0$ and $<1$, grade 2: $1-10$, grade $3:>10$ ), $\mathrm{pT} 4, \mathrm{pN} 1$, and $\mathrm{GS}>7$ were gathered in cluster $\mathrm{A}$, which was related to significantly poor RFS, suggesting an accurate risk prediction model based on 6 genes. We then analyzed the DEGs of the 6 genes in correlation with the above five factors, and MMAB and 


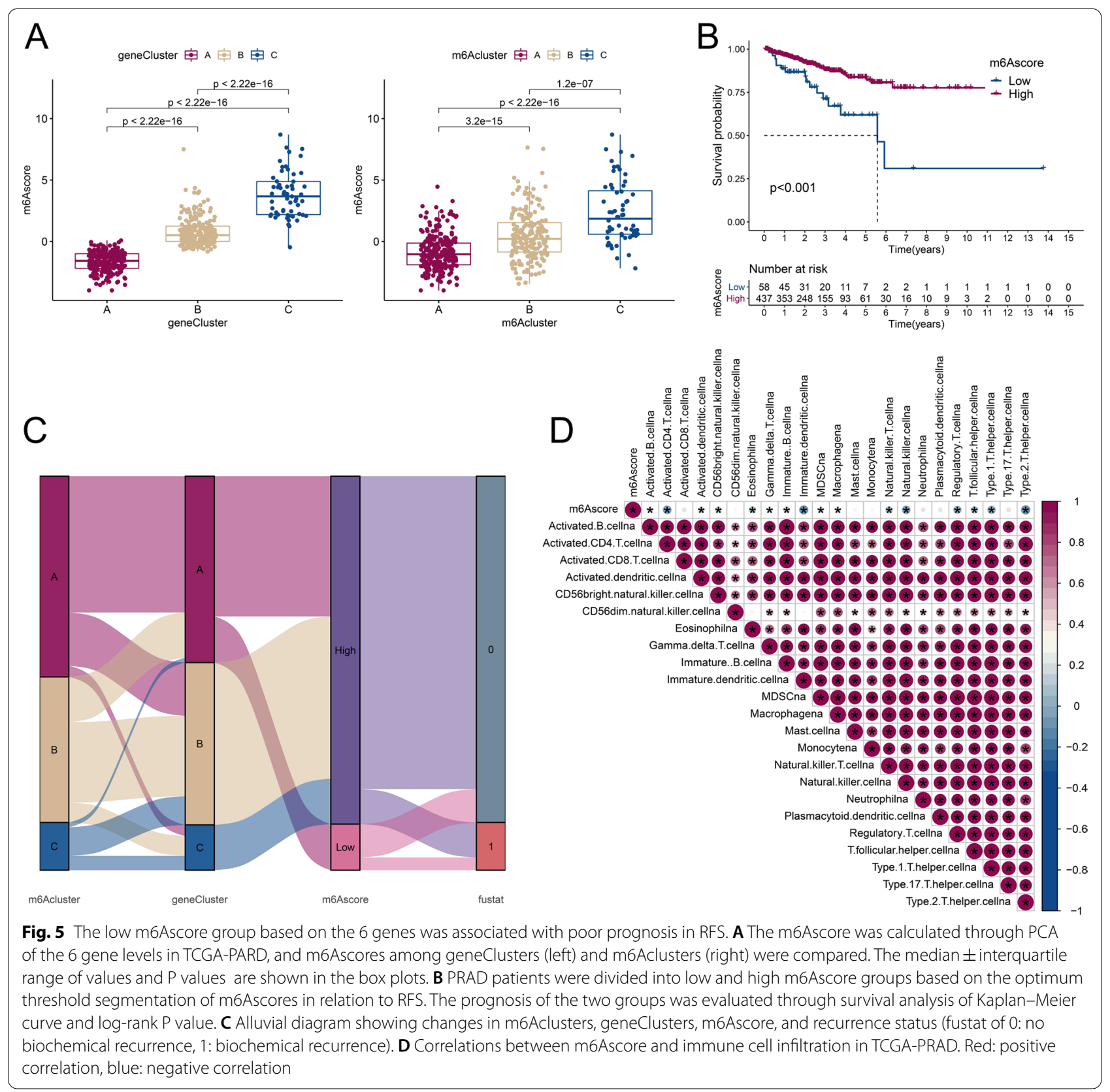

BAIAP2 were identified as intersecting genes. Levels of both were higher in $\mathrm{PCa}$ tissues than in adjacent normal prostate tissues but lower in tissues with advanced disease factors. We next verified MMAB based on different GSs of PCa tissues and adjacent normal tissues and found that its level of mRNA was elevated in PCa tissues but decreased in the GS $>7$ group, consistent with the bioinformatic analysis.

To quantitatively illustrate the m6A signature, we calculated m6Ascore according to the expression of the 6 genes in TCGA-PARD: m6Ascore correlated positively with geneCluster, and lower m6Ascore was associated with poor prognosis in RFS. Furthermore, m6Ascore was negatively correlated with 16 of 23 immune-associated cells. Thus, a potential mechanism by which the m6A signature protects against $\mathrm{PCa}$ progression is by negatively regulating immune cell infiltration. A high TMB score corresponds with tumor-related mutations and sensitivity to immunotherapy [27, 28], and Yue Zhao et al. reported that m6A modification in PCa may contribute to immunotherapy strategies [26]. In our study, the lower m6Ascore group tended to have a higher proportion of TP53 mutations $(18 \%)$, and the high m6Ascore group tended to have a higher proportion of SPOP mutations (12\%). However, 


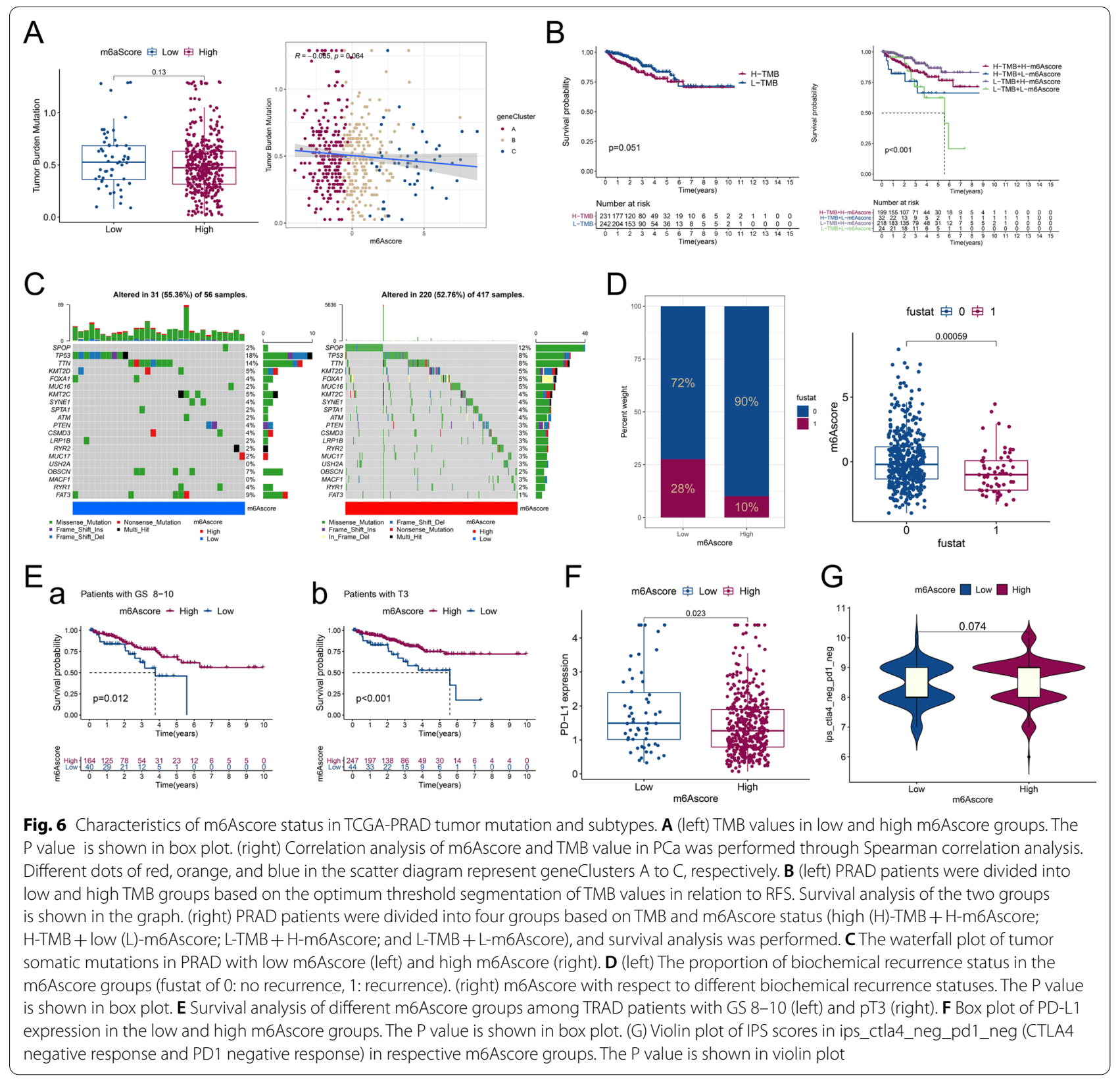

no significant correlation with TMB was found in the two m6Ascore groups in our study, and there were also no differences in PD-L1 expression or IPS score with response prediction of negative or positive PD1 and CTLA4 therapy. In summary, the results of this study do not clearly support an influence of m6A modification patterns on $\mathrm{PCa}$ immune cell infiltration and immunotherapy.

\section{Conclusions}

In this study, we comprehensively analyzed m6A regulators in TCGA-PRAD through bioinformatic analysis and tissue validation. First, we screened DEGs of m6Aclusters and found 6 genes (BAIAP2, TEX264, MMAB, JAGN1, TIMM8AP1, and IMP3), through which we divided PCa patients into three subgroups and calculated m6Ascore to construct a risk model with high predictive value for recurrence. This study may contribute to determination of the effects of $\mathrm{m} 6 \mathrm{~A}$ signaling on the progression and prognosis of PCa.

\section{Abbreviations}

PCa: Prostate cancer; ADT: Androgen deprivation therapy; CRPC: Castrationresistant prostate cancer; m6A: N6-methyladenosine; TCGA: The Cancer Genome Atlas; DEG: Differentially expressed gene; GO: Gene Ontology; KEGG: Kyoto Encyclopedia of Genes and Genomes; PCA: Principal component 
analysis; CDF: Cumulative distribution function; RFS: Recurrence-free survival; TMB:Tumor mutational burden; ssGSEA: Single-sample gene set enrichment analysis; qPCR: Quantitative real-time PCR; IPS: Immunophenoscore; GS: Gleason score; HR: Hazard ratio; Cl: Confidence interval; pT: Pathological T; pN: Pathological N; rPFS: Radiographic progression-free survival; pN: Pathological $\mathrm{N}$; OS: Overall survival.

\section{Supplementary Information}

The online version contains supplementary material available at https://doi. org/10.1186/s12935-021-02438-1.

Additional file 1: Table S1. Oligonucleotide primers of relative genes. Table S2. The expression levels of 6 DEGs in TCGA-PRAD and normal tissues. Table S3. The expression levels of 6 DEGs in TCGA-PRAD data stratified by GS. Table S4. The expression levels of 6 DEGs in TCGA-PRAD data stratified by pT. Table S5. The expression levels of 6 DEGs in TCGA-PRAD data stratified by TP53 mutation. Table S6. Abundance of each infiltrating immune cell among the three geneclusters.

Additional file 2: Fig. S1. Enrichment analysis of 74 intersecting DEGs of m6Aclusters. (A to B) Bar plot (A) and dotplot (B) of $\mathrm{GO}$ enrichment in cellular component terms, biological process terms and molecular function terms. (C to D) Bar plot (C) and dotplot (D) of KEGG enriched terms. BP: biological process; CC: cell component; MF: molecular function.

Additional file 3: Fig. S2. Characteristics of 6 DEGs of m6Aclusters in prostate cancer. (A) (top) The CNV variation frequency of 5 DEGs (TIMM8AP1 was excluded due to being a processed pseudogene and a lack of copy number data) in TCGA-PRAD. Blue dot: deletion frequency; red dot: amplification frequency. (bottom) The location of CNV alterations of 5 DEGs on 23 chromosomes. Blue dot: copy number loss, red dot: copy number gain. (B) Box plot of the expression of 6 DEGs in PCa and normal tissues. Median values \pm interquartile ranges are shown in the graph. $\mathrm{ns}$ $P>0.05 ;{ }^{*} P<0.05 ;{ }^{* *} P<0.01 ;{ }^{* * *} P<0.001$. (C) The mutation frequency of 6 DEGs in 484 TCGA-PRAD patients is shown on the waterfall plot. The columns represent individual patients, the upper bar plot shows the TMB, the right bar plot shows the proportion of each variant type, and the stacked bar plot below shows the transformed fraction of each patient.

Additional file 4: Fig. S3. Expression of 6 DEGs of m6Aclusters in PCa prognosis and different clinicopathological characteristics. (A to D) Distribution of 3-4 representative DEGs (3-4 with the lowest $P$ values) in TCGA-PRAD data stratified by GS (A), PT (B), RFS (C), and TP53 mutation (D). In the box plots, the median \pm interquartile range of values is shown in the graph, and $P$ values are shown above each pair of comparisons. (E) Prognosis network of interactions between 6 DEGs in PCa. The P values of each regulator of the prognosis are shown as circles of different sizes. Purple in the right hemisphere: risk factors for RFS; green in the right hemisphere: favorable factors for RFS. Upregulation (Up) or no significant differences (Ns) of 6 DEGs in PCa compared with normal tissues are filled with red and gray on the left hemisphere, respectively. Positive or negative correlations of 6 DEGs are linked with lines of different colors (pink: positive, blue: negative), and the correlation strength between them is shown as lines of different thicknesses.

Additional file 5: Fig. S4. Consensus clustering analysis based on 6 DEGs of m6Aclusters. (A) PCA of the transcriptome profiles of three geneClusters, showing a marked difference in the transcriptome between different m6Aclusers. Different dots of red, orange, and blue in the scatter diagram represent m6Aclusters A to C. (B) Abundance of each immune cell infiltration among three geneClusters in the boxplot. The median \pm interquartile range of values is shown in the graph. ns $P>0.05$; ${ }^{*} P<0.05$; ${ }^{* *} P<0.01$; ${ }^{* * *} P<0.001$. (C) Heatmap of KEGG enrichment analysis showing the activation states of biological pathways in distinct geneClusters. Red represents activation, and blue represents inhibition.

Additional file 6: Fig. S5. Characteristics of m6Ascore status in TCGA-PRAD tumor subtypes. (A) Survival analysis of different m6Ascore groups among TRAD patients with GS 6 (left) and GS 7 (right). (B) Survival analysis of different m6Ascore groups among TRAD patients with pT2 (left) and pT4 (right) disease. (C) Violin plot of IPS scores among ips_ctla4_neg_pd1_pos (CTLA4 negative response and PD1 positive response) (left), ips_ctla4_pos_pd1_neg (middle), and ips_ctla4_pos_ pd1_pos (right) in the respective 6 Ascore groups. The $P$ value is shown in violin plot.

\section{Acknowledgements}

We thank Beijing Chaoyang Hospital, Capital Medical University for providing samples from PCa patients.

\section{Authors' contributions}

YQ designed the study and performed the GPCR analysis. HP analyzed the data. XZ and HP provided the clinical prostate cancer samples. YQ wrote the manuscript, which was approved by all authors. All authors read and approved the final manuscript.

\section{Funding}

This study was supported by the National Natural Science Foundation of China (Grant Nos. 81772698 and 82072833 to Hao Ping) and the Open Research Fund from Beijing Advanced Innovation Center for Big Data-Based Precision Medicine, Beijing Tongren Hospital, Beihang University \& Capital Medical University (Grant No. BHTR-KFJJ-202005 to Hao Ping).

\section{Availability of data and materials}

The data and materials supporting the conclusions of this study are included within the article and its additional files.

\section{Declarations}

\section{Ethics approval and consent to participate}

Human prostate samples were provided by Beijing Tongren Hospital and Beijing Chaoyang Hospital. Ethical consent was approved by the Ethics Committee of Beijing Tongren Hospital and Beijing Chaoyang Hospital, affiliated with Capital Medical University.

\section{Consent for publication}

All authors read and approved the final manuscript for publication.

\section{Competing interests}

The authors declare that they have no competing interests.

\section{Author details}

${ }^{1}$ Department of Urology, Beijing Tongren Hospital, Capital Medical University, Beijing 100730, China. ${ }^{2}$ Beijing Advanced Innovation Center for Big Data-Based Precision Medicine, Beihang University \& Capital Medical University, Beijing Tongren Hospital, Beijing 100730, China. ${ }^{3}$ Department of Urology, Beijing Chaoyang Hospital, Capital Medical University, Beijing 100020, China.

Received: 15 October 2021 Accepted: 30 December 2021

Published online: 19 January 2022

\section{References}

1. Siegel RL, Miller KD, Jemal A. Cancer statistics, 2019. CA Cancer J Clin. 2019;69:7-34.

2. Xiao L, Tien JC, Vo J, Tan M, Parolia A, Zhang Y, Wang L, Qiao Y, Shukla S, Wang X, Zheng H, Su F, Jing X, Luo E, Delekta A, Juckette KM, Xu A, Cao X, Alva AS, Kim Y, MacLeod AR, Chinnaiyan AM. Epigenetic reprogramming with antisense oligonucleotides enhances the effectiveness of androgen receptor inhibition in castration-resistant prostate cancer. Cancer Res. 2018;78:5731-40.

3. Boccaletto P, Machnicka MA, Purta E, Piatkowski P, Baginski B, Wirecki TK, de Crécy-Lagard V, Ross R, Limbach PA, Kotter A, Helm M, Bujnicki JM. MODOMICS: a database of RNA modification pathways update. Nucleic Acids Res. 2017;2018(46):D303-d307.

4. Cantara WA, Crain PF, Rozenski J, McCloskey JA, Harris KA, Zhang X, Vendeix FA, Fabris D, Agris PF. The RNA Modification Database, RNAMDB: 2011 update. Nucleic Acids Res. 2011;39:D195-201. 
5. Meyer KD, Saletore Y, Zumbo P, Elemento O, Mason CE, Jaffrey SR. Comprehensive analysis of mRNA methylation reveals enrichment in $3^{\prime}$ UTRs and near stop codons. Cell. 2012;149:1635-46.

6. Dubin DT, Taylor RH. The methylation state of poly A-containing messenger RNA from cultured hamster cells. Nucleic Acids Res. 1975;2:1653-68.

7. Maity A, Das B. N6-methyladenosine modification in mRNA: machinery, function and implications for health and diseases. Febs j. 2016;283:1607-30.

8. Tuncel $G$, Kalkan R. Importance of $m \mathrm{~N}(6)$-methyladenosine ( $\mathrm{m}(6) \mathrm{A})$ RNA modification in cancer. Med Oncol. 2019;36:36.

9. Lin S, Choe J, Du P, Triboulet R, Gregory RI. The m(6)A Methyltransferase METTL3 Promotes Translation in Human Cancer Cells. Mol Cell. 2016:62:335-45

10. Ma XX, Cao ZG, Zhao SL. m6A methyltransferase METTL3 promotes the progression of prostate cancer via m6A-modified LEF1. Eur Rev Med Pharmacol Sci. 2020;24:3565-71.

11. Yuan Y, Du Y, Wang L, Liu X. The M6A methyltransferase METTL3 promotes the development and progression of prostate carcinoma via mediating MYC methylation. J Cancer. 2020;11:3588-95.

12. Li E, Wei B, Wang X, Kang R. METTL3 enhances cell adhesion through stabilizing integrin $\beta 1$ mRNA via an m6A-HuR-dependent mechanism in prostatic carcinoma. Am J Cancer Res. 2020;10:1012-25.

13. Cotter KA, Gallon J, Uebersax N, Rubin P, Meyer KD, Piscuoglio S, Jaffrey SR, Rubin MA. Mapping of m6A and Its Regulatory Targets in Prostate Cancer Reveals a METTL3-low Induction of Therapy Resistance. Mol Cancer Res. 2021;34:89.

14. Du C, Lv C, Feng Y, Yu S. Activation of the KDM5A/miRNA-495/YTHDF2/ m6A-MOB3B axis facilitates prostate cancer progression. J Exp Clin Cancer Res. 2020;39:223.

15. Li J, Xie H, Ying Y, Chen H, Yan H, He L, Xu M, Xu X, Liang Z, Liu B, Wang X, Zheng $X$, Xie L. YTHDF2 mediates the mRNA degradation of the tumor suppressors to induce AKT phosphorylation in N6-methyladenosinedependent way in prostate cancer. Mol Cancer. 2020;19:152.

16. Zhu K, LiY, XuY. The FTO m(6)A demethylase inhibits the invasion and migration of prostate cancer cells by regulating total m(6)A levels. Life Sci. 2021:271:119180

17. Hänzelmann S, Castelo R, Guinney J. GSVA: gene set variation analysis for microarray and RNA-seq data. BMC Bioinformatics. 2013;14:7.

18. Chen DS, Mellman I. Elements of cancer immunity and the cancerimmune set point. Nature. 2017:541:321-30.

19. Zhang B, Wu Q, Li B, Wang D, Wang L, Zhou YL. m(6)A regulator-mediated methylation modification patterns and tumor microenvironment infiltration characterization in gastric cancer. Mol Cancer. 2020;19:53.

20. Quan Y, Cui Y, Wahafu W, Liu Y, Ping H, Zhang X. MLL5a activates AR/ NDRG1 signaling to suppress prostate cancer progression. Am J Cancer Res. 2020;10:1608-29.

21. Quan Y, Zhang X, Butler W, Du Z, Wang M, Liu Y, Ping H. The role of $\mathrm{N}$-cadherin/C-Jun/NDRG1 axis in the progression of prostate cancer. Int J Biol Sci. 2021;17:3288-304.

22. Jiang Q, Chen H, Tang Z, Sun J, Ruan Y, Liu F, Sun Y. Stemness-related LncRNA pair signature for predicting therapy response in gastric cancer. BMC Cancer. 2021;21:1067.

23. Eggener SE, Scardino PT, Walsh PC, Han M, Partin AW, Trock BJ, Feng Z, Wood DP, Eastham JA, Yossepowitch O, Rabah DM, Kattan MW, Yu C, Klein EA, Stephenson AJ. Predicting 15-year prostate cancer specific mortality after radical prostatectomy. J Urol. 2011;185:869-75.

24. Kozminski MA, Tomlins S, Cole A, Singhal U, Lu L, Skolarus TA, Palapattu GS, Montgomery JS, Weizer AZ, Mehra R, Hollenbeck BK, Miller DC, He C Feng FY, Morgan TM. Standardizing the definition of adverse pathology for lower risk men undergoing radical prostatectomy. Urol Oncol. 2016;34(415):e411-416.

25. Deek MP, Van der Eecken K, Phillips R, Parikh NR, Isaacsson Velho P, Lotan TL, Kishan AU, Maurer T, Boutros PC, Hovens C, Abramowtiz M, Pollack A, Desai N, Stish B, Feng FY, Eisenberger M, Carducci M, Pienta KJ, Markowski M, Paller CJ, Antonarakis ES, Berlin A, Ost P and Tran PT. The Mutational Landscape of Metastatic Castration-sensitive Prostate Cancer: The Spectrum Theory Revisited. Eur Urol 2021;

26. Zhao Y, Sun H, Zheng J, Shao C. Analysis of RNA m(6)A methylation regulators and tumour immune cell infiltration characterization in prostate cancer. Artif Cells Nanomed Biotechnol. 2021;49:407-35.
27. Binnewies M, Roberts EW, Kersten K, Chan V, Fearon DF, Merad M, Coussens LM, Gabrilovich DI, Ostrand-Rosenberg S, Hedrick CC, Vonderheide RH, Pittet MJ, Jain RK, Zou W, Howcroft TK, Woodhouse EC, Weinberg RA, Krummel MF. Understanding the tumor immune microenvironment (TIME) for effective therapy. Nat Med. 2018;24:541-50.

28. Quail DF, Joyce JA. Microenvironmental regulation of tumor progression and metastasis. Nat Med. 2013;19:1423-37.

29. Van Neste L, Groskopf J, Grizzle WE, Adams GW, DeGuenther MS, Kolettis PN, Bryant JE, Kearney GP, Kearney MC, Van Criekinge W, Gaston SM. Epigenetic risk score improves prostate cancer risk assessment. Prostate. 2017;77:1259-64

30. Natesan R, Aras S, Effron SS, Asangani IA. Epigenetic Regulation of Chromatin in Prostate Cancer. Adv Exp Med Biol. 2019;1210:379-407.

31. Desrosiers R, Friderici K, Rottman F. Identification of methylated nucleosides in messenger RNA from Novikoff hepatoma cells. Proc Natl Acad Sci U S A. 1974;71:3971-5.

32. Alarcón CR, Lee H, Goodarzi H, Halberg N, Tavazoie SF. N6-methyladenosine marks primary microRNAs for processing. Nature. 2015;519:482-5.

33. Patil DP, Chen CK, Pickering BF, Chow A, Jackson C, Guttman M, Jaffrey SR. m(6)A RNA methylation promotes XIST-mediated transcriptional repression. Nature. 2016;537:369-73.

34. Chen Y, Pan C, Wang X, Xu D, Ma Y, Hu J, Chen P, Xiang Z, Rao Q, Han X. Silencing of METTL3 effectively hinders invasion and metastasis of prostate cancer cells. Theranostics. 2021;11:7640-57.

35. Cotter KA, Gallon J, Uebersax N, Rubin P, Meyer KD, Piscuoglio S, Jaffrey $\mathrm{SR}$, Rubin MA. Mapping of m(6)A and Its Regulatory Targets in Prostate Cancer Reveals a METTL3-Low Induction of Therapy Resistance. Mol Cancer Res. 2021;19:1398-411.

36. Zhang Q, Luan J, Song L, Wei X, Xia J, Song N. Malignant Evaluation and Clinical Prognostic Values of M6A RNA Methylation Regulators in Prostate Cancer. J Cancer. 2021;12:3575-86.

37. Ji G, Huang C, He S, Gong Y, Song G, Li X, Zhou L. Comprehensive analysis of $\mathrm{m} 6 \mathrm{~A}$ regulators prognostic value in prostate cancer. Aging (Albany NY). 2020;12:14863-84.

38. Ou-Yang S, Liu JH, Wang QZ. Expression patterns and a prognostic model of $\mathrm{m}(6) \mathrm{A}$-associated regulators in prostate adenocarcinoma. Biomark Med. 2020;14:1663-77.

39. Wang J, Lin H, Zhou M, Xiang Q, Deng Y, Luo L, Liu Y, Zhu Z, Zhao Z. The $\mathrm{m} 6 \mathrm{~A}$ methylation regulator-based signature for predicting the prognosis of prostate cancer. Future Oncol. 2020;16:2421-32.

40. Wu Q, Xie X, Huang Y, Meng S, Li Y, Wang H, Hu Y. N6-methyladenosine RNA methylation regulators contribute to the progression of prostate cancer. J Cancer. 2021;12:682-92.

41. Liu B, Jiang HY, Yuan T, Luo J, Zhou WD, Jiang QQ, Wu D. EnzalutamideInduced Upregulation of PCAT6 Promotes Prostate Cancer Neuroendocrine Differentiation by Regulating miR-326/HNRNPA2B1 Axis. Front Oncol. 2021;11:650054.

42. Jiang M, Lu Y, Duan D, Wang H, Man G, Kang C, Abulimiti K, Li Y. Systematic Investigation of mRNA N (6)-Methyladenosine Machinery in Primary Prostate Cancer. Dis Markers. 2020;2020:8833438.

43. Cheng Y, Li L, Qin Z, Li X, Qi F. Identification of castration-resistant prostate cancer-related hub genes using weighted gene co-expression network analysis. J Cell Mol Med. 2020;24:8006-17.

44. Singh AN, Sharma N. Quantitative SWATH-based proteomic profiling for identification of mechanism-driven diagnostic biomarkers conferring in the progression of metastatic prostate cancer. Front Oncol. 2020;10:493.

45. Peng Z, Andersson K, Lindholm J, Dethlefsen O, Pramana S, Pawitan Y, Nistér M, Nilsson S, Li C. Improving the prediction of prostate cancer overall survival by supplementing readily available clinical data with gene expression levels of IGFBP3 and F3 in formalin-fixed paraffin embedded core needle biopsy material. PLOS ONE. 2016;11:e0145545.

46. Prager AJ, Peng CR, Lita E, McNally D, Kaushal A, Sproull M, Compton K, Dahut WL, Figg WD, Citrin D, Camphausen KA. Urinary aHGF, IGFBP3 and OPN as diagnostic and prognostic biomarkers for prostate cancer. Biomark Med. 2013;7:831-41.

47. Qie Y, Nian X, Liu X, Hu H, Zhang C, Xie L, Han R, Wu C, Xu Y. Polymorphism in IGFBP3 gene is associated with prostate cancer risk: an updated meta-analysis. Onco Targets Ther. 2016;9:4163-71.

48. Zhang X, Wang D, Liu B, Jin X, Wang X, Pan J, Tu W, Shao Y. IMP3 accelerates the progression of prostate cancer through inhibiting PTEN expression in a SMURF1-dependent way. J Exp Clin Cancer Res. 2020;39:190. 
49. Szarvas T, Tschirdewahn S, Niedworok C, Kramer G, Sevcenco S, Reis H, Shariat SF, Rübben H, vom Dorp F. Prognostic value of tissue and circulating levels of IMP3 in prostate cancer. Int J Cancer. 2014;135:1596-604.

50. Ikenberg K, Fritzsche FR, Zuerrer-Haerdi U, Hofmann I, Hermanns T, Seifert H, Müntener M, Provenzano M, Sulser T, Behnke S, Gerhardt J, Mortezavi A, Wild P, Hofstädter F, Burger M, Moch H, Kristiansen G. Insulin-like growth factor II mRNA binding protein 3 (IMP3) is overexpressed in prostate cancer and correlates with higher Gleason scores. BMC Cancer. 2010;10:341.

\section{Publisher's Note}

Springer Nature remains neutral with regard to jurisdictional claims in published maps and institutional affiliations.

- fast, convenient online submission

- thorough peer review by experienced researchers in your field

- rapid publication on acceptance

- support for research data, including large and complex data types

- gold Open Access which fosters wider collaboration and increased citations

- maximum visibility for your research: over $100 \mathrm{M}$ website views per year

At BMC, research is always in progress.

Learn more biomedcentral.com/submissions 